\title{
Exercise, or exercise and diet for the management of polycystic ovary syndrome: a systematic review and meta-analysis
}

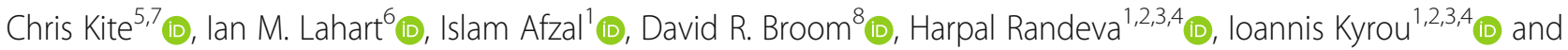
James E. Brown ${ }^{1,5^{*}}$

\begin{abstract}
Background: Typically, management of PCOS focuses on lifestyle changes (exercise and diet), aiming to alleviate symptoms, and lower the associated risk of type 2 diabetes and cardiovascular disease. Our objective was to analyse evidence on the effectiveness of exercise in the management of PCOS, when compared to (i) usual care, (ii) diet alone, and (iii) exercise combined with diet, and also exercise combined with diet, compared to (i) control or usual care and (ii) diet alone.

Methods: Relevant databases were searched (June 2017) with no time limit for trial inclusion. Eligible trials employed a randomised or quasi-randomised design to measure the chronic effects of exercise, or exercise and diet in women with PCOS.

Results: Searches returned 2390 articles; of those, 27 papers from 18 trials were included. Results are presented as mean difference (MD) and 95\% confidence intervals (95\% Cl). Compared with control, exercise had a statistical effect on change from baseline fasting insulin ( $\mathrm{MD}-2.44 \mu \mathrm{lU} / \mathrm{mL}, 95 \% \mathrm{Cls}-4.24$ to -0.64 ; very low-quality evidence), HOMA-IR $(-0.57,-0.99$ to -0.14 ; very low-quality evidence), total cholesterol $(-5.88 \mathrm{mg} / \mathrm{dL},-9.92$ to -1.83 ; low-quality evidence), LDL cholesterol $(-7.39 \mathrm{mg} / \mathrm{dL},-9.83$ to -4.95 ; low-quality evidence), and triglycerides ( $-4.78 \mathrm{mg} / \mathrm{dL},-7.52$ to -2.05 ; low-quality evidence). Exercise also improved $\mathrm{VO}_{2}$ max $(3.84 \mathrm{ml} / \mathrm{kg} / \mathrm{min}, 2.87$ to 4.81$)$, waist circumference $(-2.62 \mathrm{~cm},-4.13$ to -1.11$)$, and body fat percentage $(-1.39 \%,-2.61$ to -0.18$)$ when compared with usual care. No effect was found for change value systolic/ diastolic blood pressure, fasting glucose, HDL cholesterol (all low-quality evidence), or waist-to-hip ratio. Many favourable change score findings were supported by post-intervention value analyses: fasting insulin $(-2.11 \mu \mathrm{UU} / \mathrm{mL},-3.49$ to -0.73$)$, total cholesterol $(-6.66 \mathrm{mg} / \mathrm{dL},-11.14$ to -2.17$)$, LDL cholesterol $(-6.91 \mathrm{mg} / \mathrm{dL},-12.02$ to -1.80$)$, and $\mathrm{VO}_{2}$ $\max (5.01 \mathrm{ml} / \mathrm{kg} / \mathrm{min}, 3.48$ to 6.54$)$. Statistically lower BMI $\left(-1.02 \mathrm{~kg} / \mathrm{m}^{2},-1.81\right.$ to -0.23$)$ and resting heart rate $(-3.26$ beats $/ \mathrm{min}-4.93$ to -1.59$)$ were also revealed in post-intervention analysis. Subgroup analyses revealed the greatest improvements in overweight/obese participants, and more outcomes improved when interventions were supervised, aerobic in nature, or of a shorter duration. Based on limited data, we found no differences for any outcome between the effects of exercise and diet combined, and diet alone. It was not possible to compare exercise vs diet or exercise and diet combined vs diet.

\footnotetext{
* Correspondence: J.E.P.Brown@aston.ac.uk

${ }^{1}$ Aston Medical Research Institute, Aston Medical School, Aston University,

Birmingham B4 7ET, UK

${ }^{5}$ School of Life and Health Sciences, Cell and Tissue Biomedical Research

Group, Aston University, Aston Triangle, Birmingham B4 7ET, UK

Full list of author information is available at the end of the article
}

(c) The Author(s). 2019 Open Access This article is distributed under the terms of the Creative Commons Attribution 4.0 International License (http://creativecommons.org/licenses/by/4.0/), which permits unrestricted use, distribution, and reproduction in any medium, provided you give appropriate credit to the original author(s) and the source, provide a link to the Creative Commons license, and indicate if changes were made. The Creative Commons Public Domain Dedication waiver (http://creativecommons.org/publicdomain/zero/1.0/) applies to the data made available in this article, unless otherwise stated. 
(Continued from previous page)

Conclusion: Statistically beneficial effects of exercise were found for a range of metabolic, anthropometric, and cardiorespiratory fitness-related outcomes. However, caution should be adopted when interpreting these findings since many outcomes present modest effects and wide Cls, and statistical effects in many analyses are sensitive to the addition/removal of individual trials. Future work should focus on rigorously designed, well-reported trials that make comparisons involving both exercise and diet.

Systematic review registration: This systematic review was prospectively registered on the Prospero International Prospective Register of Systematic Reviews (CRD42017062576)

Keywords: Polycystic ovary syndrome, Exercise, Physical activity, Diet, Cardiovascular risk, Insulin resistance, Cardiorespiratory fitness

\section{Background}

Polycystic ovary syndrome (PCOS) is the most common endocrinopathy in reproductive-aged women, affecting 6-21\% (depending on the applied diagnostic criteria) of this population worldwide [1-3]. PCOS is characterised by hyperandrogenism and/or chronic anovulation which can manifest with a range of symptoms (e.g., hirsutism, acne, oligomenorrhea, and infertility) [4] and is associated with increased risk of cardiometabolic disease, including hypertension, dyslipidaemia, insulin resistance (IR), and type 2 diabetes mellitus (T2DM) [5]. Moreover, PCOS is linked to increased psychological morbidity [e.g., increased risk of stress, depression, low self-esteem, poor body image, and reduced health-related quality of life (HRQoL)] [6, 7]. The exact PCOS aetiology is unknown, but increased adiposity is considered pivotal [8]. Indeed, almost $90 \%$ of women with PCOS are overweight or obese and even moderate weight loss (e.g., 5\%) may result in clinically meaningful improvements in hyperandrogenism and menstrual regularity [9-13]. Also, women with PCOS often have more severe IR than weight-matched women without PCOS [14, 15], whilst their increased susceptibility to obesity [16] may further exacerbate IR and the accompanying metabolic $[17,18]$ and reproductive $[10,19]$ dysfunctions. As such, women with PCOS exhibit increased risk of impaired glucose tolerance and T2DM regardless of weight and age [20].

As there is currently no curative treatment for PCOS, management of overweight/obese women with PCOS focuses on weight loss through regular exercise and diet, aiming to alleviate its clinical manifestations and lower the related risk of T2DM and cardiovascular disease (CVD) [21]. Considering the benefits of exercise interventions in other IR populations independent of weight loss [22-24], incorporating moderate-intensity exercise in PCOS treatment may be particularly favourable. Existing evidence supports this; although most exercise trials in women with PCOS show little or no weight loss [5], exercise can have favourable effects on IR, body fat distribution, and CVD risk in these patients [25]. As the number of studies investigating the effects of exercise and diet in PCOS is increasing, it is important to summarise this body of evidence in order to better inform clinical practice. Therefore, this systematic review aims to analyse the evidence on the effectiveness of exercise compared to (i) control or usual care, (ii) diet alone, and (iii) exercise combined with diet, as well as the effectiveness of exercise combined with diet compared to (i) control or usual care and (ii) diet alone.

\section{Methods}

This systematic review was prospectively registered on the Prospero International Prospective Register of Systematic Reviews (CRD42017062576) and is reported based on the guidelines of the Preferred Reporting Items for Systematic Reviews and Meta-Analyses (PRISMA) statement [26].

\section{Search methods for identification of studies}

Table 1 presents the eligibility criteria for inclusion in this systematic review. Only trials with women of

Table 1 Eligibility criteria for including studies in this systematic review

Inclusion criteria:

1. Study design: randomised controlled trials and quasi-randomised controlled trials.

2. Types of participants: reproductive-aged women with a diagnosis of polycystic ovary syndrome (PCOS) based on the National Institute of Health (NIH) diagnostic criteria (1990), the Rotterdam ESHRE/ASRM (2003) diagnostic criteria or the AE-PCOS Criteria (2006). We also included trials where the PCOS diagnosis had been verified by a general practitioner or specialist clinician.

3. Comparators: exercise vs usual care/control, exercise combined with diet vs usual care/control, exercise combined with diet vs diet only. Exercise combined with diet vs exercise only, exercise vs diet, exercise combined with pharmaceutical vs pharmaceutical.

4. All outcomes; expected outcomes included: primary outcomes, such as blood pressure, fasting blood glucose, insulin and lipid concentrations; and secondary outcomes, such as body mass index, cardiorespiratory fitness, testosterone, free androgen index and health-related quality of life measures.

Exclusion criteria:

1. Study design: case studies, cross sectional and non-randomised controlled trials.

2. Types of participants: males, adolescent females, post-menopausal women, women without PCOS

3. Comparators: women with PCOS vs healthy controls, pharmaceutical vs exercise, pharmaceutical vs diet, diet vs diet, surgical vs any other condition. 
reproductive age who had received a PCOS diagnosis were eligible for inclusion. Eligible trials employed a randomised or quasi-randomised experimental (intervention) design to measure the chronic effects of exercise or exercise and diet in women with PCOS. We defined exercise as a potential disruption to homeostasis by muscle activity that is either exclusively, or in combination, concentric, eccentric, or isometric [27]. Accordingly, we accepted all methods of exercise training, including continuous aerobic exercise (e.g., walking, jogging, or cycling); high-intensity interval training; resistance training; flexibility training; and yoga, Tai Chi, and Pilates. Trials were eligible if they had a pre-post design that compared at least two conditions, using either within-subject crossover design or between-subject comparison to a control/alternative treatment group. Studies, which included follow-up testing at least 1 month after completion of the intervention, were also included.

The databases searched were CENTRAL (in the Cochrane Library), PubMed, CINAHL, SCOPUS, EMBASE (via Web of Science), SportDiscus (via EBSCOhost), and PsycINFO (via OvidSP). A search algorithm was developed for PubMed (Additional file 1: Table S1), which was then modified for each database searched.

Searches were completed in June 2017 with no time limit specified for trial inclusion. Only fully published, peer-reviewed papers were included, whereas grey literature was not eligible. No language restrictions were placed on the search.

Initial searches were completed by one reviewer (CK), duplicate records were removed before title, and abstracts were screened independently by two reviewers (CK and IML). Subsequently, full-text eligibility screening was completed independently by two reviewers (CK and IML). Any disagreements on eligibility were resolved by discussion, whilst any unresolved disagreements by arbitration from a third reviewer (DRB).

Where multiple publications for the same trial were retrieved, they were linked together, and the earliest paper of the trial was used as the primary reference. The earliest paper was used as the reference only, and data were extracted from all papers with the most comprehensive available data included for each outcome. Data were extracted from eligible studies, and a summary of these findings are presented in Table 2. Trial data were combined in meta-analyses using Review Manager (RevMan 5.3.5, Copenhagen, Denmark).

All trial outcomes were considered for inclusion following the search, but the primary outcomes were those linked to CVD risk (e.g., blood pressure, lipids, and glucose). Secondary outcomes were cardiorespiratory fitness (CRF), anthropometric measures, androgen levels, pro-inflammatory markers, and psychosocial outcomes.

\section{Assessment of risk of bias in included studies}

The Cochrane Collaboration's tool for assessing risk of bias was used; and six specific domains (sequence generation, allocation concealment, blinding, incomplete outcome data, selective outcome reporting, and any other sources of bias) were assessed. Two reviewers (CK and IA) assessed risk of bias, and a third reviewer (IML) arbitrated conflicts not due to assessor error. The Cochrane Handbook recommendations [28] were followed, and each bias parameter was graded as either high, low, or unclear risk. We judged studies with $>20 \%$ of data missing as at a high risk of attrition bias. We considered studies with between-group baseline differences that may affect the outcome, less than $75 \%$ adherence in the intervention group, and contamination in the control group (i.e., control group participants engaged in exercise), as high risk of 'other sources of bias' [29]. In exercise trials, it is difficult to blind participants and researchers to the interventions resulting in a high risk of performance bias being made; this should not infer that the methodological quality of the trial is poor, but rather that the inevitable bias related to lack of blinding has been acknowledged by the reviewers. A risk of bias table is presented in Additional file 1: Table S2 and risk of bias summarised in the results (Fig. 2; Additional file 2: Figure S1).

\section{Strategy for data synthesis}

Where data from $\geq 2$ trials were available, pooled intervention effect estimates and their $95 \%$ confidence intervals (CIs) are presented. Meta-analytical methods for involving continuous outcomes assume that data are normally distributed; hence, data were excluded from the meta-analysis when they were clearly skewed, or results were reported with median and range values, and non-parametric tests used for analysis.

Outcomes across each trial were presented as continuous data and, based on the Cochrane Handbook's recommendations [30], the random-effects method for meta-analysis was utilised to combine data [31]. Mean \pm standard deviation (SD) data for either change from baseline to post-intervention or immediately post-intervention values were combined in a meta-analysis. The RevMan calculator was used to convert standard errors, CIs, or $t$ values to SD where necessary. A priori, the analysis was based on change from baseline scores as it removed a component of between-person variability [30]; immediately, postintervention analysis was also included so as to nullify the effect of selective reporting, but also to better indicate whether there was a treatment effect regardless of baseline values. Mean difference (MD) was used where trials reported the same outcome using the same scale. Where scales varied, units of measurement were converted to the most common measure [e.g., fasting insulin (FI) converted from $\mathrm{pmol} / \mathrm{L}$ to 


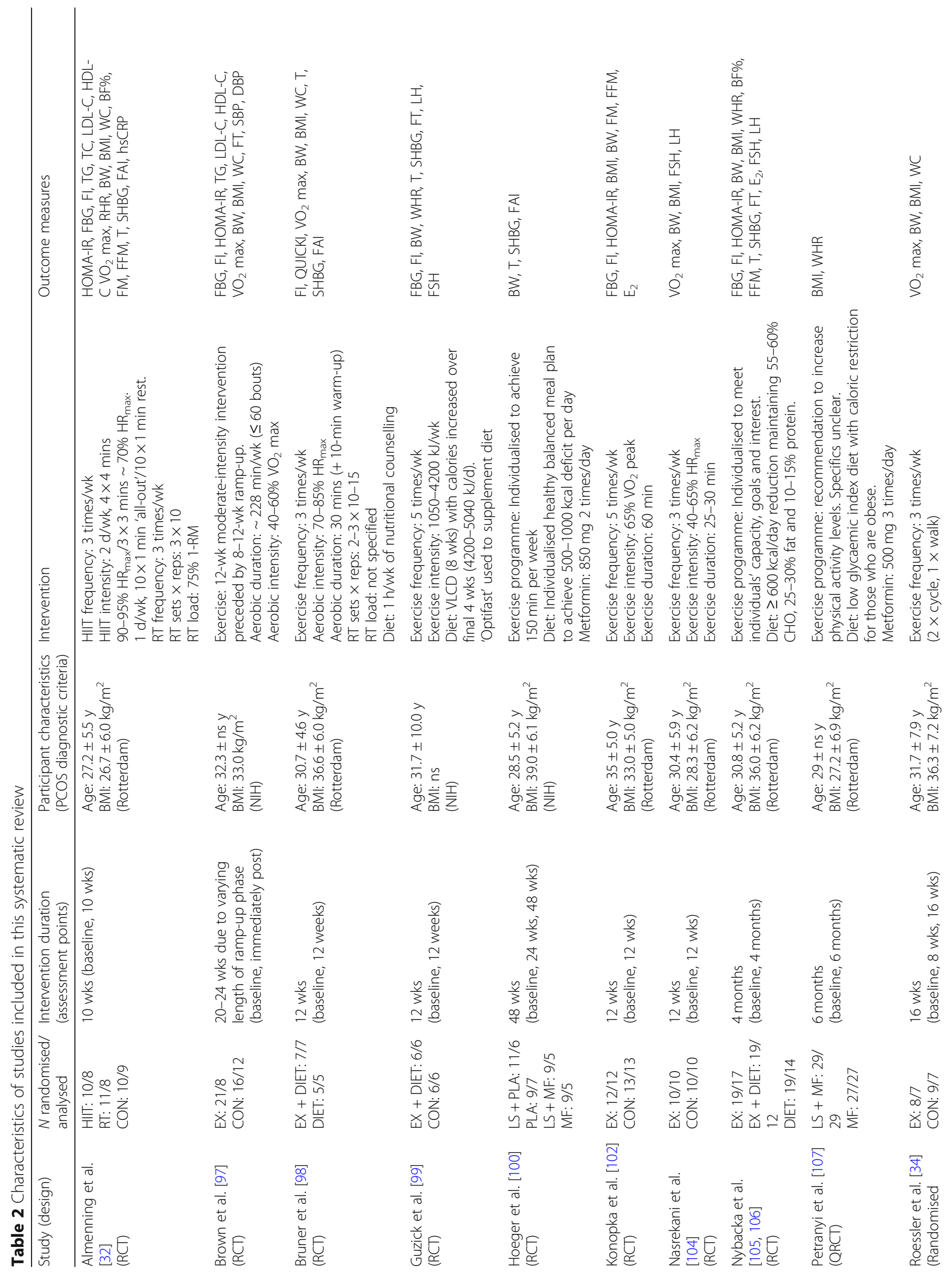


Kite et al. Systematic Reviews

(2019) 8:51

Page 5 of 28

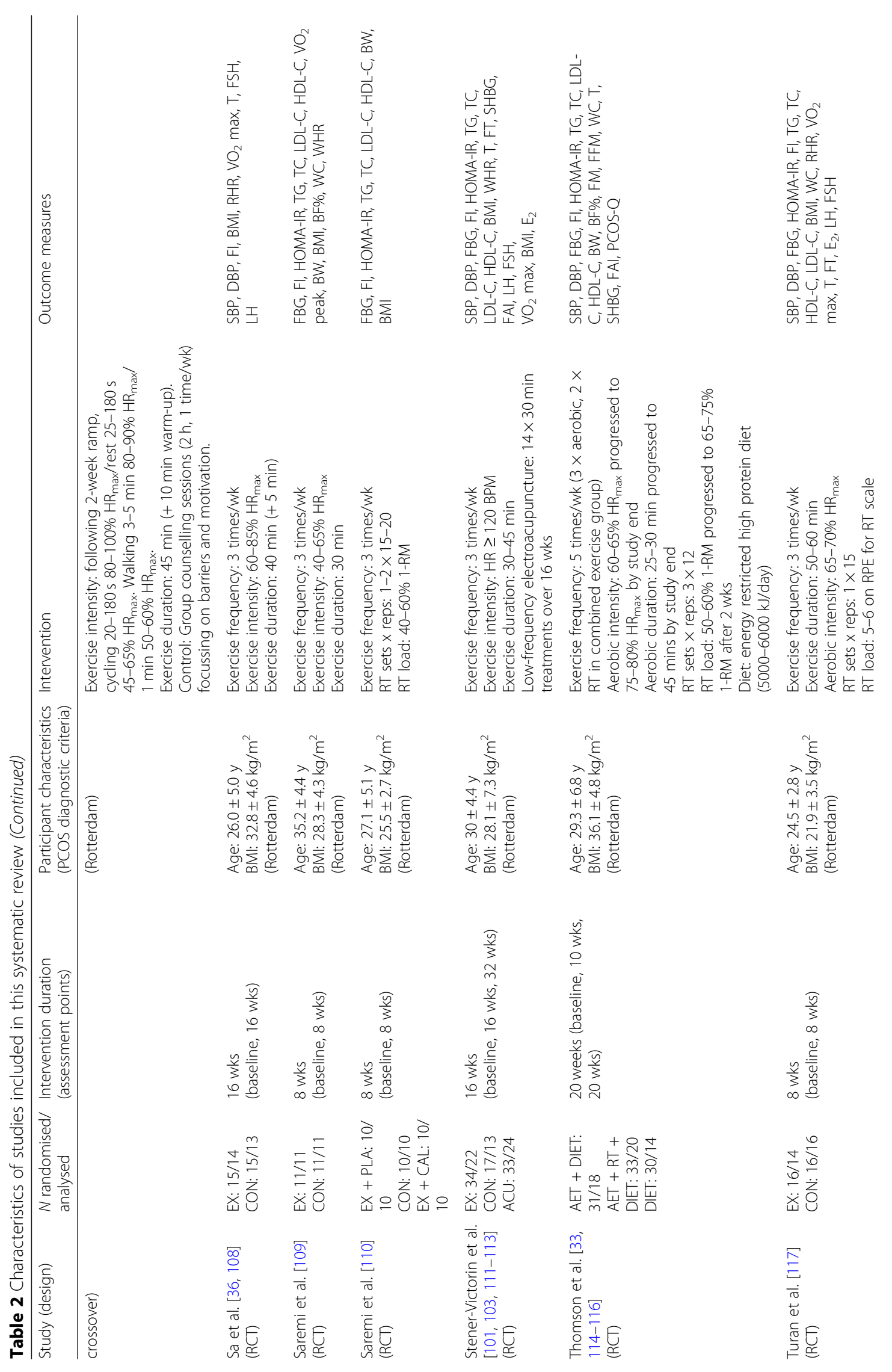




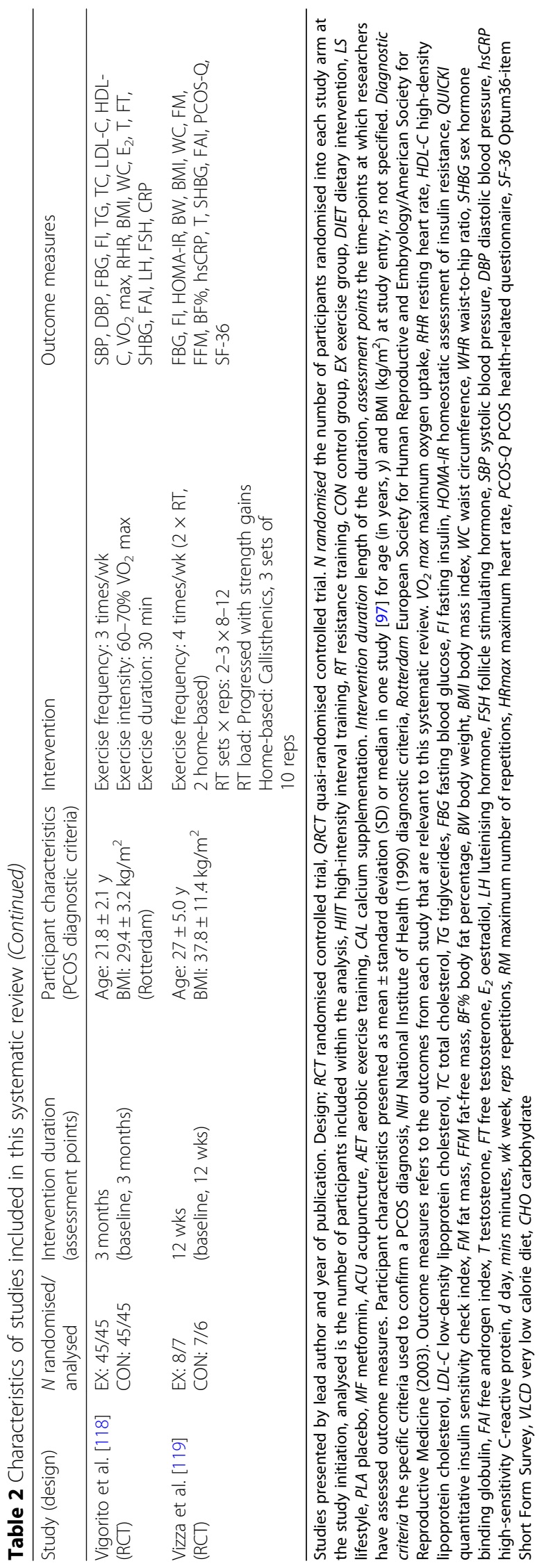


$\mu \mathrm{IU} / \mathrm{mL}]$. If this was not possible, standardised mean difference (SMD) was used. Immediately, post-intervention values were also assessed, and their data reported. If trials contained more than one eligible intervention arm [32, 33], outcome data from both groups were combined using methods recommended by Deeks et al. [30]. If an included trial used a crossover design [34], then only data up to the point of crossover were used.

We used the Grades of Recommendation, Assessment, Development, and Evaluation (GRADE) approach [35] to assess the quality of the evidence for our primary outcomes: systolic and diastolic blood pressure, blood glucose, FI, homeostatic model assessment of insulin resistance index (HOMA-IR), total cholesterol (TC), low-density lipoprotein cholesterol (LDL-C), high-density lipoprotein cholesterol (HDL-C), and triglycerides. GRADEpro GDT software was used to develop the 'Summary of findings' table, and two review authors (IML and CK) graded the quality of the evidence for each outcome. We did not downgrade based on lack of blinding alone due to difficulties of blinding participants and exercise supervising personnel. We downgraded based on risk of bias only if a lack of blinding was accompanied by additional high risks of bias (e.g., selection bias and incomplete outcome reporting).

\section{Investigation of heterogeneity}

The $I^{2}$ statistic was used to evaluate the heterogeneity of results for each outcome, across studies. Although not a measure of absolute heterogeneity, the $I^{2}$ describes the percentage of variability in the point estimates that is due to heterogeneity rather than sampling error [30]. We interpreted heterogeneity as $0-40 \%$ 'might not be important', 30-60\% 'may represent moderate heterogeneity', 50-90\% 'may represent substantial heterogeneity', and $75-90 \%$ 'considerable heterogeneity' [30]. The importance of the observed $I^{2}$ value depends on the magnitude and direction of effects, as well as the strength of evidence for heterogeneity. Visual inspection of forest plots was completed, and statistical heterogeneity assumed if there was little or no overlap of CIs for the results of individual studies. When evidence of at least substantial heterogeneity was present, its source was investigated by study population groups-the trial that represented the largest outlier was removed from the analysis and the $I^{2}$ was re-evaluated. If heterogeneity was not reduced, it was also assessed in subgroup analyses.

\section{Assessment of reporting biases}

To investigate publication bias, if there were $\geq 10$ trials included in an analysis, we used a funnel plot to explore the possibility of small study effects-a tendency for smaller studies to report larger beneficial effects. This was only completed for one outcome (BMI) because when there are fewer studies $(<10)$, the power of tests is too low to distinguish chance from real asymmetry [30].

\section{Subgroup analysis}

Where there were data from $\geq 2$ studies, analyses of subgroups was conducted. Study characteristics analysed were body mass index (BMI) upon study entry (BMI $\leq$ $24.9 \mathrm{~kg} / \mathrm{m}^{2}, 25.0-29.9 \mathrm{~kg} / \mathrm{m}^{2}$ or $\left.\geq 30.0 \mathrm{~kg} / \mathrm{m}^{2}\right)$, intervention type (aerobic exercise, resistance training, or combination of the two), intervention duration ( $\leq 12$ weeks or $>12$ weeks), and intervention delivery format (supervised, unsupervised, or mixed delivery). Outcome data were separated by subgroup, and subtotal summary statistics were presented. The available data were insufficient to complete three of the sub-analyses (exercise intensity, combined treatments, and behaviour change components) outlined in the original protocol, but findings have been reported qualitatively where available.

\section{Sensitivity analysis}

Sensitivity analyses were completed on outcomes where an effect was observed to assess the effect of removing small sample size studies $(n<30$ total participants) and those with high overall bias risk. Due to the nature of the interventions, performance and detection bias were removed from the reviewers' judgement. All studies exhibited at least one domain where risk of bias was unclear, so only those with at least one domain where risk of bias was deemed to be high were removed.

\section{Results}

\section{Description of included studies Search results}

In total, 2390 articles were identified from the database searches; we were also sent one additional article after requesting further information from another author [36]. After removing duplicates, 1908 articles were screened for eligibility based on title and abstract. A total of 87 full-text articles were retrieved for detailed eligibility evaluation, and 60 of these were excluded [37-96] with reasons detailed in Fig. 1 and Additional file 1: Table S3.

Following exclusion, 27 met the inclusion criteria [32-34, 36, 97-104, 106-119]. However, these publications were based on 18 trials, since four trials had multiple publications, namely Stener-Victorin et al. [111] four additional papers [101, 103, 112, 113]; Thomson et al. [33] three additional publications [114-116]; Nybacka et al. [105] one additional publication [106]; and Sa et al. [108] one additional publication [36].

One study was excluded from the meta-analysis [97] because data were reported as median and range values (attempts to contact the author were unsuccessful). 


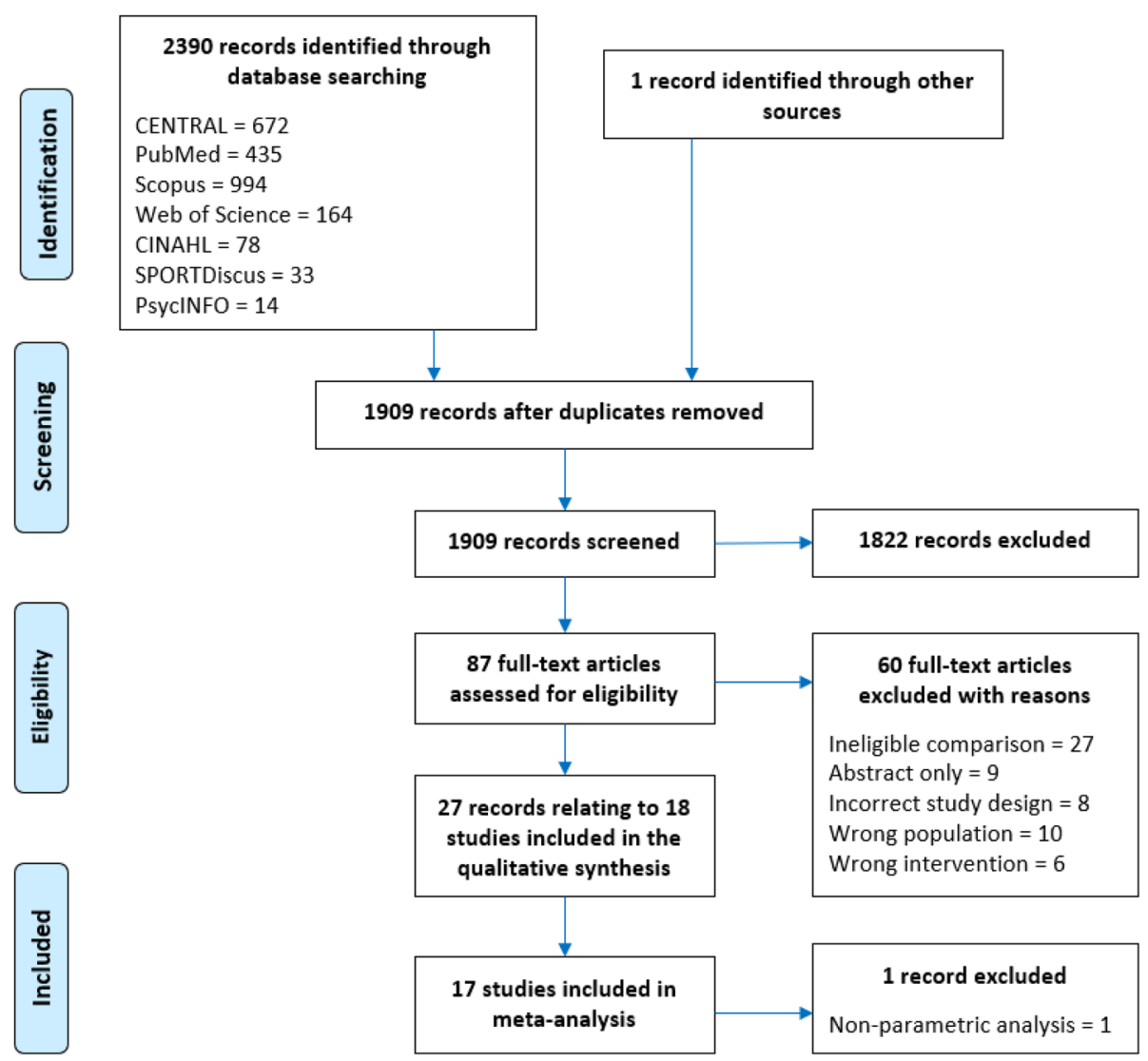

Fig. 1 Preferred Reporting Items for Systematic Reviews and Meta-analyses (PRISMA) flow diagram

\section{Eligible studies design and attrition}

Of the 18 included trials, 16 were randomised controlled clinical trials (RCTs), whilst one trial had a quasi-RCT [107] and another a randomised crossover [34] design.

Twelve trials compared exercise to usual care or minimal intervention [32, 34, 97, 102, 104, 108-111, 117-119]. Three trials each compared combined exercise and diet with diet only [33, 98, [105]], and exercise and diet combined with usual care $[99,100,107]$. Only one trial [105]investigated exercise versus diet and exercise versus exercise and diet combined. The total number of participants included within the trials were 758 (exercise/intervention, $n$ = 230; control, $n=257$; combined treatment arms, $n=174$; and diet alone, $n=54$ ). In addition, 43 participants were included in ineligible arms, i.e., pharmacological arm [100, 107, 110] and low-frequency electroacupuncture [111].

Eight trials (44\%) did not report any attrition [98, 99, 102, 104, 107, 109, 110, 118]. Where reported, attrition ranged from $6 \%$ [117] to $50 \%$ [33] with a median value of $19.5 \%$; five trials (28\%) reported attrition over $20 \%$ $[32,33,97,100,105]$. Reasons for exercise dropouts included non-exercise related injury [32-34, 97, 119], pregnancy [32-34, 100, 119], time [33, 34, 97], work/ family commitments $[32,33,119]$, personal reasons [33,
$105,108,111]$, medical grounds [97, 105, 111], and relocation [33]. Two trials excluded participants because adherence to intervention was $<75 \%$ [117] or failure to comply with study requirements [33].

\section{Participant characteristics of included studies}

Participant characteristics are presented in Table 2. Included trials used a range of criteria to diagnose PCOS as presented in Additional file 1: Table S4, with three trials [97, 99, 100] using the NIH diagnostic criteria [120], whereas 14 [32-34, 98, 102, 104, 105, 107-111, 117, 118] used the Rotterdam consensus criteria [121]. One trial confirmed the PCOS diagnosis via participants' general practitioner/specialist [119], but criteria used were unclear. No trials specified use of the AE-PCOS definition [122].

Participants with T2DM, fasting hyperglycaemia, or glucose intolerance were explicitly excluded in nine trials (50\%) [33, 34, 97, 98, 102, 109, 111, 117, 118], and nine trials also excluded participants with any diagnosed CVD [33, 34, 98, 99, 109, 111, 117-119]. Another prerequisite in seven trials (39\%) was the activity status of participants upon enrolment, namely a sedentary lifestyle and no recent participation in an exercise intervention had to be apparent [32, 33, 97, 102, 104, 109, 119]. 


\section{Intervention and comparison details}

Fourteen trials (74\%) assessed the effectiveness of an exercise-only intervention and six trials (32\%) assessed a combined exercise and dietary intervention. Moreover, 14 trials $(74 \%)$ included intervention arms consisting of aerobic exercise only, and a further three (16\%) combined aerobic exercise with resistance training [33, 98, 117]. Of those incorporating aerobic exercise $(n=17$, 94\%), 11 trials (61\%) specified either walking, brisk walking, or jogging [32-34, 97-99, 104, 105, 108, 109, 111] and seven (39\%) incorporated static cycling either on its own or as part of a wider intervention [32, 34, 97, 98, $102,111,118]$. A trial each incorporated elliptical training [97], step training [117] or swimming [105]. Five trials $(28 \%)[32,97,98,105,111]$ allowed participants to self-select modality from those listed above, whereas two trials $[100,107]$ allowed participants to self-select a modality but without stating the choices. Three trials (16\%) had arms that were resistance training only [32, 110, 119]. However, in one trial, the type of exercise was unclear [107].

The modal training session frequency was three per week in 10 trials (56\%) [32, 33, 98, 104, 108-111, 117, 118]. Five sessions per week were prescribed in three (17\%) trials [33, 99, 102], whereas in another trial [119] four sessions per week were set. Of the remaining four trials, one specified a weekly physical activity (PA) time target of $150 \mathrm{~min}$ per week [100], one trial set an exercise dose of $14 \mathrm{kcal} / \mathrm{kg} /$ week [97], and two did not specify training frequency or volume [105, 107].

Eight trials (44\%) set aerobic exercise intensity using a percentage of the maximum heart rate $\left(\mathrm{HR}_{\max }\right)$ [32-34, $98,104,108,109,117]$ or maximal oxygen uptake $\left(\mathrm{VO}_{2}\right.$ $\max )[97,102,118]$. One trial specified that heart rate (HR) was set at $\geq 120$ beats/min [111]. Three trials using resistance training prescribed intensity based on a percentage of 1-repetition maximum (either 40-60\% [110] or $50-75 \%[32,33])$. One resistance training intervention set intensity using a rate of perceived exertion of 56 out of 10 [117]. Six trials did not specify the intensity of the intervention [98-100, 105, 107, 119]. Four trials increased the intensity as the intervention progressed [10-104, 106-110].

Eleven trials (61\%) prescribed session durations of 1 hour or less $(\leq 30 \mathrm{~min}[32,34,104,109,118],>30-60$ $\min [97,102,108,111,119]$, or $20-30$ to $45 \mathrm{~min}$ [33]). Only one trial consisted of training sessions of $>60 \mathrm{~min}$ [98]. Hoeger and colleagues [100] specified $150 \mathrm{~min}$ as a weekly target, whereas another trial used a target distance of 10 miles per week [99]. Four trials did not specify timings for their intervention $[11,105,107,110]$.

In ten trials (56\%), participants were fully supervised in all exercise sessions [34, 98, 102, 104, 105, 108-110, $117,118]$, whilst two $(11 \%)$ used a mixed approach with some supervised sessions $[32,119]$ and one $(6 \%)$ was unsupervised with support provided weekly by telephone [111]. The remaining five trials (26\%) did not report supervision status.

Six trials (33\%) incorporated a dietary component. Five of these trials (28\%) specified either a daily caloric target [33, 99], a reduced caloric intake [105, 107], or an individualised caloric deficit [100]. The other of these trials [98] used weekly nutritional counselling sessions to educate participants on a range of nutritional topics.

Thirteen trials $(72 \%)$ had a control arm (Table 2) [32, 34, 96. 98, 101, 103, 107, 109-111, 117-119]. Three of these trials offered participants the intervention $[34,99]$ or a 1-month gym membership [32] upon completion of the trial (wait-list control). Three of the remaining trials $(17 \%)$ used a diet-only arm as their comparison group $[33,98,105]$, one trial used a placebo [100], and another used metformin treatment only [107].

\section{Characteristics of the outcome measures}

All studies assessed participants at baseline and immediately post-intervention (Table 2), whilst two trials incorporated an additional midway assessment $[33,100]$, one trial added a follow-up assessment 16 weeks post-intervention [111], and another trial assessed at baseline, crossover, and immediately post-intervention [34]. No post-intervention follow-up analysis was possible due to lack of studies.

Seven trials (39\%) stated the sample size calculation methods [32, 97, 105, 108-111, 118], although only five $(28 \%)$ of those reported the outcome upon which their calculations were based [32, 97, 108, 111]. The primary outcomes (used in sample size calculation) were HOMA-IR [32], $\mathrm{VO}_{2}$ peak [108], total testosterone [111], insulin sensitivity [97], and BMI [105]. Only three trials stated recruitment targets [32, 108, 111]; all three trials achieved their sample size calculated target. The outcomes included in each trial are provided in Table 2.

\section{Assessment of risk of bias in included studies}

The authors' risk of bias judgements are presented in the risk of bias graph (Fig. 2), whilst further details are included in Additional file 2: Figure S1 and Additional file 1: Table S2.

Only four trials (22\%) were judged to have a low risk of selection bias, using appropriate methods to generate their randomisation sequence and conceal allocation [32, $97,117,119]$. One trial was judged to be at high risk of selection bias [108] because five participants were allocated to the control group based upon their geographical location. The remaining trials were judged to have an unclear risk of selection bias due to insufficient reporting of sequence generation or allocation concealment methods. Due to the nature of the interventions, all 


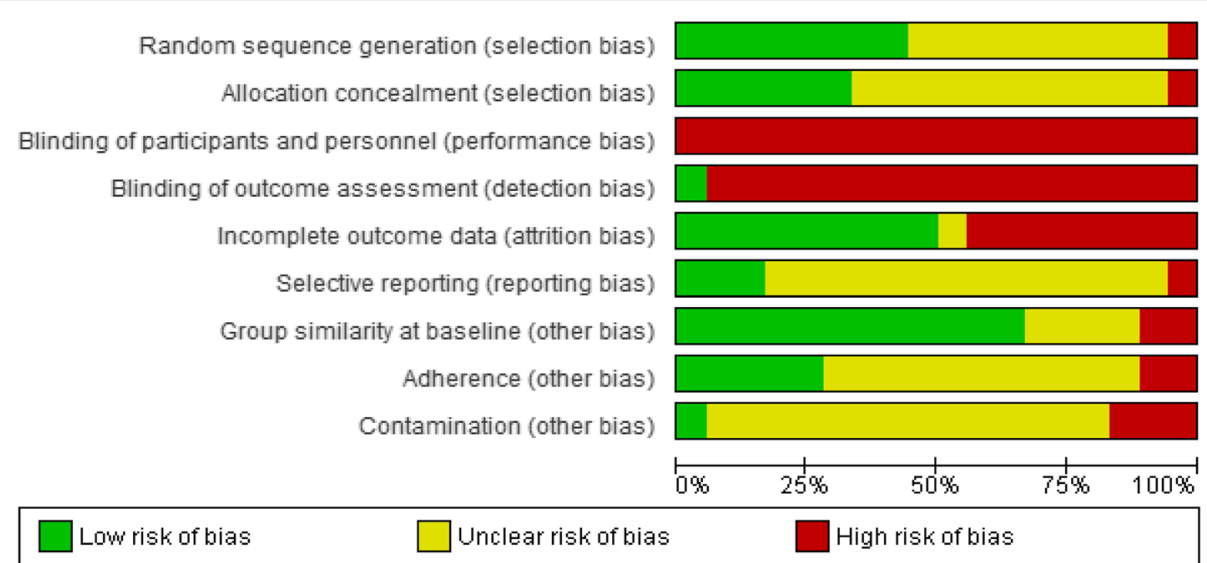

Fig. 2 Review of authors' judgement of each methodological quality item from the Cochrane Risk of Bias tool, presented as a percentage across all 18 included studies

trials were judged to be at a high risk for performance bias. Only one trial had a low risk for detection bias [118]; the remaining trials were judged to be at a high risk of this bias due to not stating whether outcome assessors were blinded to participant allocation. One trial [32] used an independent, and blinded, assessor for evaluation of only one outcome (flow-mediated dilation).

Eight trials (44\%) were judged to be high risk for attrition bias because participant withdrawal rates were $>20 \%$ $[33,97,100,105,111]$, incomplete data due to lab error [98], inappropriate handling of missing data (i.e., last observation carried forward) [119], and only a subset of participants completing hyperinsulinaemic-euglycaemic clamp testing [102]. A prospective protocol document or trial registration was available only for three trials, thus making it difficult to judge whether all intended outcomes had been reported. The remaining 14 trials $(78 \%)$ were judged to have an unclear risk of reporting bias, and one trial [108] was judged to be high risk due to incomplete reporting of results.

Eleven trials (61\%) had low risk of bias based upon statistical similarities between groups at baseline [32, 34, 98-100, 102, 105, 108, 111, 114, 117]. Of the high-risk trials, one [119] had participants in the intervention group with less favourable adiposity and body composition versus control. Similarly, another trial [97] had an older exercise group that was less hyperandrogenic and hirsute, and had lower levels of CRF and higher BMI, plasma lipids, and IR levels compared with controls.

Adherence was reported in seven trials (39\%) [32, $34,97,111,117-119]$, with a median of $90 \%$ adherence, ranging from 67\% [34] to 103\% [111]. Two of the trials $(11 \%)$ reported intervention adherence below the $75 \%$ threshold outlined in the "Methods" section [34, 119]. Five trials $(28 \%)$ were judged to have a low risk of adherence bias (adherence $\geq 75 \%$ )
[32, 97, 111, 117, 118]. Finally, most trials $(n=14$, $78 \%$ ) were deemed to have unclear risk of contamination bias due to lack of reporting. Only one trial [118] had a low risk of contamination bias as the control group did not increase PA $>4 \mathrm{MET} / \mathrm{h} /$ week [123], whereas three trials (17\%) had a high risk of contamination as it was reported that comparison groups had either engaged in treatment [34, 111] or control groups had not received their allocated intervention [108].

\section{Effects of interventions: Exercise versus control}

Due to data availability, a meta-analysis was possible only for three comparisons: (1) exercise versus control, (2) exercise and diet combined versus control, and (3) exercise and diet combined versus diet only.

Eleven trials were included in the exercise versus control meta-analysis as presented in Table $3[32,34,102$, 104, 108-111, 117-119].

\section{Primary outcomes Blood pressure}

Four eligible trials (158 participants) assessed changes in blood pressure. We found no significant effect of exercise on systolic blood pressure (SBP) or diastolic blood pressure (DBP) for either change scores or post-intervention values compared with control (Table 3). We rated the result of both SBP and DBP as low-quality evidence due to imprecision (small number of participants, and a null and appreciable effect were included in the 95\% CI for the MD), and high or unclear risk of selection bias, detection bias, reporting bias, attrition bias, and contamination (see Table 4; Summary of findings for primary outcomes).

In subgroup analyses (Additional file 1: Table S5), we only found effects of supervised interventions (MD: - 
Table 3 Effect estimates and heterogeneity for change from baseline to post-intervention scores and immediately post-intervention values, for all outcomes analysed in the exercise versus control comparison

\begin{tabular}{|c|c|c|c|c|c|c|c|c|c|c|c|}
\hline \multirow[t]{2}{*}{ Outcome } & \multirow[t]{2}{*}{ References } & \multicolumn{5}{|c|}{ Change from baseline } & \multicolumn{5}{|c|}{ Immediately post-intervention values } \\
\hline & & $N$ & $\mathrm{MD}$ & $\begin{array}{l}\text { Lower 95\% } \\
\mathrm{Cl}\end{array}$ & $\begin{array}{l}\text { Upper 95\% } \\
\text { Cl }\end{array}$ & $\begin{array}{l}P^{2} \\
(\%)\end{array}$ & $N$ & $\mathrm{MD}$ & $\begin{array}{l}\text { Lower 95\% } \\
\mathrm{Cl}\end{array}$ & $\begin{array}{l}\text { Upper 95\% } \\
\mathrm{Cl}\end{array}$ & $\begin{array}{l}p^{2} \\
(\%)\end{array}$ \\
\hline $\mathrm{SBP}(\mathrm{mmHg})$ & {$[101,108,117,118]$} & 158 & -2.93 & -7.06 & 1.20 & 50 & 158 & 2.02 & -6.82 & 10.86 & 87 \\
\hline $\mathrm{DBP}(\mathrm{mmHg})$ & {$[101,108,117,118]$} & 158 & -2.19 & -5.23 & 0.85 & 46 & 158 & -0.82 & -3.49 & 1.84 & 31 \\
\hline $\mathrm{FBG}(\mathrm{mg} / \mathrm{dL})$ & {$[32,100,101,107,109,110,117-119]$} & 263 & -1.08 & -2.47 & 0.30 & 16 & 238 & -1.69 & -4.35 & 0.97 & 37 \\
\hline $\mathrm{FI}(\mu \mathrm{IU} / \mathrm{mL})$ & {$[32,100,101,107,109,110,117-119]$} & 263 & $-4^{* *}$ & -4.24 & -0.64 & 91 & 238 & $-{ }^{-} .11^{* *}$ & -3.49 & -0.73 & 40 \\
\hline HOMA-IR & {$[32,100,101,107,109,110,117,119]$} & 173 & $-\overline{-}^{*} 7^{* *}$ & -0.99 & -0.14 & 87 & 148 & -0.22 & -0.80 & 0.36 & 69 \\
\hline $\mathrm{TC}(\mathrm{mg} / \mathrm{dL})$ & {$[32,101,108-110,117,118]$} & 225 & - & -9.92 & -1.83 & 35 & 225 & $-\overline{6.35^{* *}}$ & -10.76 & -1.95 & 0 \\
\hline LDL-C (mg/dL) & {$[32,101,108-110,117,118]$} & 225 & $\begin{array}{l}- \\
7.39 * * *\end{array}$ & -9.83 & -4.95 & 0 & 225 & - & -11.66 & -1.70 & 0 \\
\hline $\mathrm{HDL}-\mathrm{C}(\mathrm{mg} / \mathrm{dL})$ & {$[32,101,108-110,117,118]$} & 225 & 0.29 & -1.46 & 2.04 & 52 & 225 & 1.87 & -1.59 & 5.33 & 65 \\
\hline TG (mg/dL) & {$[32,101,108-110,117,118]$} & 225 & $-78^{* * *}$ & -7.52 & -2.05 & 3 & 225 & -1.97 & -7.36 & 3.42 & 18 \\
\hline $\mathrm{VO}_{2} \max (\mathrm{ml} / \mathrm{kg} / \mathrm{min}) \boldsymbol{\Lambda}$ & {$[32,100,103,107,109,118]$} & 229 & $3.84^{* * *}$ & 2.87 & 4.81 & 17 & 184 & $5.01^{* * *}$ & 3.48 & 6.54 & 42 \\
\hline RHR (bpm) & {$[32,101,117,118]$} & 156 & -2.65 & -5.55 & 0.25 & 51 & 156 & $3.26^{* * *}$ & -4.93 & -1.59 & 0 \\
\hline BMI $\left(\mathrm{kg} / \mathrm{m}^{2}\right)$ & $\begin{array}{l}{[32,34,100,101,103,107,109,110,117} \\
118,119]\end{array}$ & 331 & -0.49 & -1.04 & 0.06 & 66 & 272 & $-\overline{1.02^{* *}}$ & -1.81 & -0.23 & 0 \\
\hline Body Mass (kg) & {$[32,34,101,103,109,110,119]$} & 139 & -1.25 & -3.27 & 0.76 & 33 & 128 & -0.48 & -4.86 & 3.91 & 0 \\
\hline WC $(\mathrm{cm})$ & {$[32,34,108,109,117-119]$} & 221 & $-2^{* * * *}$ & -4.13 & -1.11 & 53 & 221 & -2.33 & -5.23 & 0.58 & 15 \\
\hline WHR & {$[101,118]$} & 101 & -0.03 & -0.08 & 0.02 & 0 & 101 & -0.04 & -0.08 & 0.01 & 19 \\
\hline Body Fat (\%) & {$[32,109,119]$} & 60 & $-1.39^{*}$ & -2.61 & -0.18 & 30 & 60 & -3.28 & -7.39 & 0.83 & 22 \\
\hline Fat Mass (kg) & {$[32,101,119]$} & 63 & -1.70 & -3.93 & 0.53 & 70 & 38 & 5.14 & -14.39 & 24.68 & 65 \\
\hline $\mathrm{FFM}(\mathrm{kg}) \boldsymbol{\Lambda}$ & {$[32,101,119]$} & 63 & 0.46 & -0.89 & 1.81 & 58 & 38 & 4.99 & -7.31 & 17.28 & 75 \\
\hline Testosterone (nmol/L) & {$[32,101,117-119]$} & 203 & -0.09 & -0.24 & 0.06 & 0 & 169 & -0.08 & -0.35 & 0.19 & 37 \\
\hline SHBG (nmol/L) & {$[32,101,118,119]$} & 173 & 7.51 & -8.01 & 23.04 & 89 & 139 & 4.03 & -18.57 & 26.63 & 66 \\
\hline Free $T(\mathrm{pg} / \mathrm{mL})$ & {$[101,117]$} & 74 & -0.43 & -1.74 & 0.88 & 76 & 41 & 0.33 & -0.10 & 0.77 & 0 \\
\hline FAl & {$[32,101,118,119]$} & 139 & 0.24 & -0.55 & 1.04 & 0 & 139 & 0.68 & -1.09 & 2.44 & 46 \\
\hline FG & {$[101,118]$} & 135 & -0.63 & -2.08 & 0.81 & 0 & 101 & -0.75 & -2.03 & 0.54 & 0 \\
\hline Oestradiol (pmol/L) & {$[100,101,117,118]$} & 190 & -13.94 & -54.53 & 26.64 & 65 & 120 & 0.27 & -11.27 & 11.80 & 0 \\
\hline DHEA-S $(\mu \mathrm{mol} / \mathrm{L})$ & {$[32,101]$} & 70 & -0.60 & -1.58 & 0.39 & 0 & 36 & -0.20 & -1.87 & 1.46 & 0 \\
\hline LH (IU/L) & {$[101,104,117,118]$} & 185 & -0.30 & -2.54 & 1.95 & 72 & 151 & -0.66 & -2.39 & 1.06 & 43 \\
\hline FSH (IU/L) & {$[101,104,117,118]$} & 185 & 0.23 & -0.08 & 0.53 & 0 & 151 & -0.01 & -0.40 & 0.37 & 0 \\
\hline LH/FSH ratio & {$[101,117]$} & 41 & -0.02 & -0.38 & 0.33 & 0 & 41 & 0.32 & -0.22 & 0.86 & 37 \\
\hline PG (nmol/L) & {$[102,118]$} & 115 & -0.72 & -2.53 & 1.09 & 74 & - & - & - & - & - \\
\hline Prolactin (ng/mL) & {$[104,118]$} & 110 & -0.05 & -0.71 & 0.61 & 0 & 110 & 0.20 & -0.27 & 0.68 & 0 \\
\hline hsCRP (mg/L) & {$[32,119]$} & 38 & -0.41 & -1.19 & 0.37 & 0 & 38 & 0.67 & -1.31 & 2.65 & 0 \\
\hline $\mathrm{AMH}(\mathrm{ng} / \mathrm{mL})$ & {$[32,109,110]$} & 67 & -0.67 & -1.65 & 0.32 & 0 & 67 & 0.48 & -1.89 & 2.84 & 0 \\
\hline Adiponectin $(\mu \mathrm{g} / \mathrm{mL})$ & {$[32,101]$} & 70 & -0.20 & -1.04 & 0.64 & 0 & - & - & - & - & - \\
\hline
\end{tabular}

Effect estimates are reported as mean differences (MD) and 95\% confidence intervals, between exercise and usual care groups. Heterogeneity reported using $I^{2}$ statistic

Key: $95 \% \mathrm{Cl}$ 95\% confidence intervals, SBP systolic blood pressure, DBP diastolic blood pressure, $F B G$ fasting blood glucose, $F I$ fasting insulin, $H O M A-I R$ homeostatic model of assessment - insulin resistance, $T C$ total cholesterol, $L D L-C$ low-density lipoprotein cholesterol, $H D L-C$ high-density lipoprotein cholesterol, TG triglycerides, $R H R$ resting heart rate, $B M I$ body mass index, WC waist circumference, WHR waist-to-hip ratio, FFM fat-free mass, $S H B G$ sex hormone binding globulin, Free $T$ free testosterone, FAl free androgen index, FG Ferriman-Gallwey score, DHEA-S dehydroepiandrosterone sulfate, $L H$ luteinising hormone, FSH follicle stimulating hormone, $P G$ progesterone, $h S C R P$ high-sensitivity $C$-reactive protein, $A M H$ anti-Müllerian hormone. $N$ number or participants included within analysis

$\triangle$ Positive values favour exercise over control.

Study only included in the change from baseline analysis

Statistically significant effects denoted by ${ }^{*} P \leq 0.05 ;{ }^{*} P \leq 0.01 ;{ }^{* *} P \leq 0.001$ 


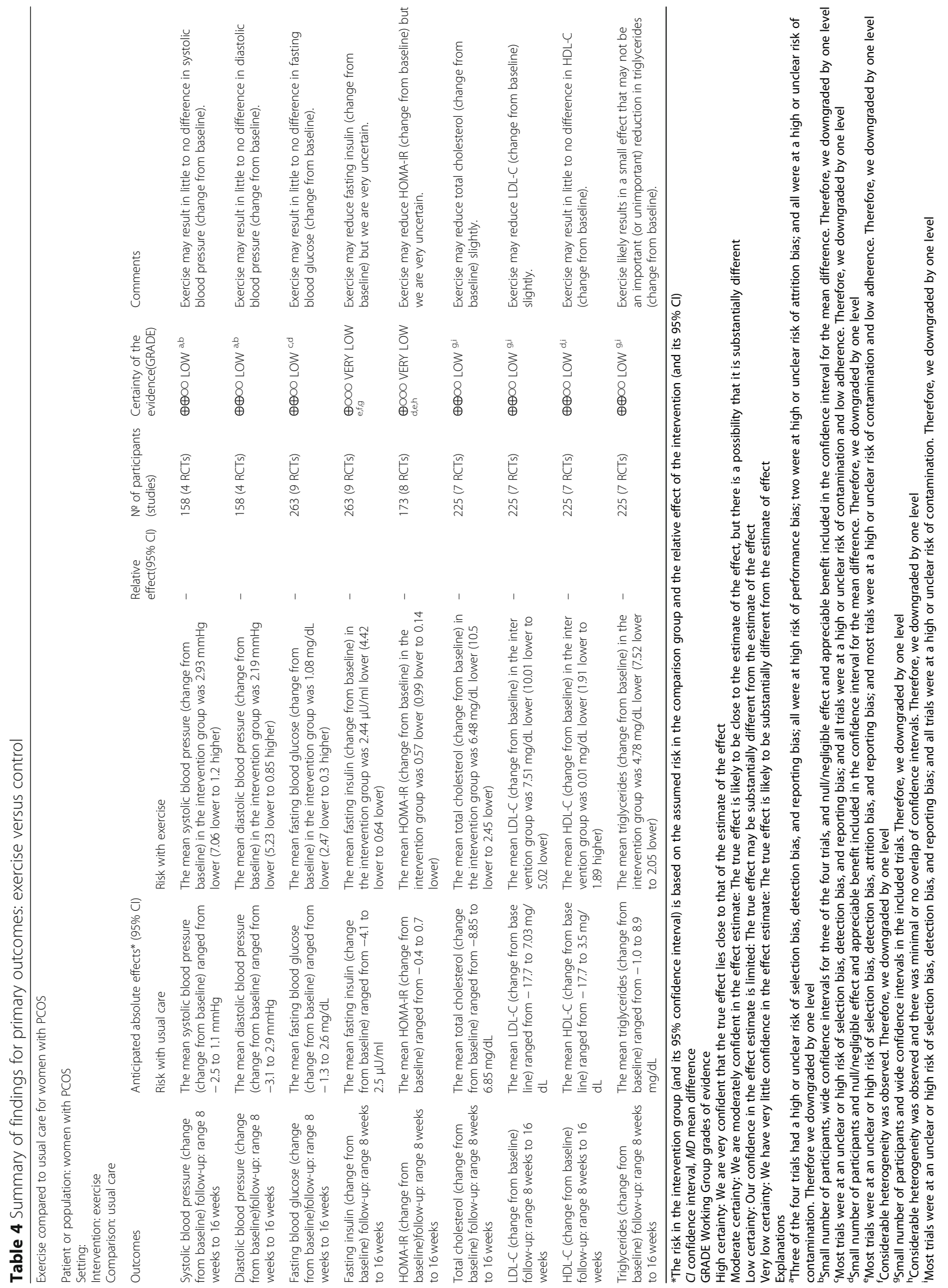


$4.42 \mathrm{mmHg}$, 95\% CI: -8.32 to -0.51 ; 3 trials, 147 participants, $I^{2}=31 \%$ ) on the SBP change compared with control. No effects were found in the subgroup analysis of SBP post-intervention values or in any DBP subgroup analysis.

\section{Fasting blood glucose}

Based on data from nine trials (263 participants), we found no effect of exercise on fasting blood glucose (FBG) change or absolute post-intervention values compared with control (Table 3). There was also no effect of exercise for any of the subgroup analyses presented in Additional file 1: Table S5. We rated the result as low-quality evidence due to an unclear or high-risk of selection, detection, and reporting bias, contamination, low adherence, small number of participants, and a null or negligible effect and appreciable benefit included in the confidence interval for the mean difference (Table 4).

\section{Fasting insulin}

Meta-analysis of nine trials (263 participants) revealed a favourable effect of exercise on the change of FI values from baseline compared with control (MD $-2.44 \mu \mathrm{IU} /$ $\mathrm{mL}, 95 \% \mathrm{CI}-4.24$ to -0.64 ; Fig. 3), but with evidence of considerable heterogeneity $\left(I^{2}=91 \%\right)$. Similarly, statistically significant lowering effects of exercise versus control were found for FI post-intervention values (MD $-2.11 \mu \mathrm{IU} / \mathrm{mL}, 95 \% \mathrm{CI}-3.49$ to -0.73 ; 8 trials, 238 participants, $\left.I^{2}=40 \%\right)$. Applying GRADE, we rated the result as very low-quality (Table 4 ) evidence due to unclear or high-risk randomisation or allocation procedures, lack of blinding, high rate of incomplete outcome data, unclear reporting of outcomes and contamination, low adherence, considerable heterogeneity in the effects in individual studies, small number of participants, and wide confidence interval for the mean difference.

In sensitivity analyses, the observed effect of exercise on FI change from baseline remained when only trials with larger sample sizes ( $n \geq 30$ total participants) (MD $-1.09 \mu \mathrm{IU} / \mathrm{mL}, 95 \% \mathrm{CI}-1.64$ to -0.53 ; 2 trials, 120 participants, $I^{2}=7 \%$ ) and studies with a low risk of bias (MD $-3.18 \mu \mathrm{IU} / \mathrm{mL}, 95 \% \mathrm{CI}-5.63$ to $-0.74 ; 187$ participants, 5 trials, $I^{2}=95 \%$ ) were included. Likewise, post-intervention FI effects remained when small trials (MD $-1.73 \mu \mathrm{IU} / \mathrm{mL}, 95 \% \mathrm{CI}-3.00$ to $-0.47 ; 2$ trials, 160 participants, $I^{2}=5 \%$ ) and trials with a high risk of bias (MD $-2.10 \mu \mathrm{IU} / \mathrm{mL}, 95 \% \mathrm{CI}-3.04$ to $-1.17 ; 5$ trials, 187 participants, $I^{2}=0 \%$ ) were removed.

To identify the potential source of heterogeneity in the FI change analysis, when the greatest outlier [110] was removed, the $I^{2}$ statistic was reduced to a level that may not be important (18\%) and the effect was maintained (MD $-1.54 \mu \mathrm{IU} / \mathrm{mL}, 95 \% \mathrm{CI}-2.36$ to -0.71$)$. The

\begin{tabular}{|c|c|c|c|c|c|c|c|c|c|c|c|}
\hline \multirow{2}{*}{ Study or Subgroup } & \multicolumn{3}{|c|}{ Exercise } & \multicolumn{3}{|c|}{ Control } & \multirow[b]{2}{*}{ Weight } & \multirow{2}{*}{$\begin{array}{c}\text { Mean Difference } \\
\text { IV, Random, } 95 \% \mathrm{Cl}[\text { ulU/mL] }\end{array}$} & \multirow{2}{*}{\multicolumn{2}{|c|}{$\begin{array}{c}\text { Mean Difference } \\
\text { IV, Random, } 95 \% \mathrm{CI}[\mathrm{ulU} / \mathrm{mL}]\end{array}$}} & Risk of Bias \\
\hline & Mean [ulU/mL] & SD [ulU/mL] & Total & Mean [ulU/mL] & $\mathrm{SD}[\mathrm{ulU} / \mathrm{mL}]$ & Total & & & & & $\triangle B C D E F G H$ \\
\hline \multicolumn{12}{|c|}{ 1.1.1 Fasting Insulin (change from baseline) } \\
\hline Sa 2015 & -2.51 & 4.98 & 14 & -4.05 & 13.02 & 13 & $4.2 \%$ & $1.54[-6.00,9.08]$ & $\longrightarrow$ & & $\oplus \ominus \oplus ? \theta$ \\
\hline Saremi 2013 & -4.7 & 5.02 & 11 & -0.82 & 4.11 & 11 & $9.2 \%$ & $-3.88[-7.71,-0.05]$ & $\ldots$ & & (4?? ? ? \\
\hline Almenning 2015 & -2.15 & 2.51 & 16 & 2.5 & 5.3339 & 9 & $9.5 \%$ & $-4.65[-8.35,-0.95]$ & $\longrightarrow$ & & \\
\hline Stener-Victorin 2009 & -1.6 & 2.37 & 5 & -1.4 & 3.01 & 6 & $10.6 \%$ & $-0.20[-3.38,2.98]$ & $\rightarrow$ & & $8+\odot \odot$ \\
\hline Vizza 2016 & -1 & 3.5992 & 7 & 1 & 1.5723 & 6 & $11.1 \%$ & $-2.00[-4.95,0.95]$ & $\Rightarrow$ & & ? ? ? \\
\hline Konopka 2015 & -1.4 & 1.7321 & 12 & 1 & 5.0478 & 13 & $11.1 \%$ & $-2.40[-5.31,0.51]$ & $\rightarrow-1$ & & $\odot \odot ? ?$ \\
\hline Vigorito 2007 & -1.8 & 3.4247 & 45 & 0.2 & 5.2637 & 45 & $13.5 \%$ & $-2.00[-3.83,-0.17]$ & $\Rightarrow$ & & ๑? ? ? \\
\hline Saremi 2016 & -3.97 & 1.1 & 10 & 1.09 & 0.58 & 10 & $15.3 \%$ & $-5.06[-5.83,-4.29]$ & $=$ & & ? ? ? ? ? \\
\hline Turan 2015 & -0.8 & 0.3742 & 14 & 0.2 & 0.8 & 16 & $15.6 \%$ & $-1.00[-1.44,-0.56]$ & & & $\odot ? \odot \odot ?$ \\
\hline Subtotal $(95 \% \mathrm{Cl})$ & & & 134 & & & 129 & $100.0 \%$ & $-2.44[-4.24,-0.64]$ & $\bullet$ & & \\
\hline \multicolumn{12}{|c|}{$\begin{array}{l}\text { Heterogeneity: } \text { Tau }^{2}=5.37 ; \mathrm{Chi}^{2}=85.60, \mathrm{df}=8(\mathrm{P}<0.00001) ; \mathrm{I}^{2}=91 \% \\
\text { Test for overall effect: } Z=2.66(P=0.008)\end{array}$} \\
\hline \multicolumn{12}{|c|}{ 1.1.2 Fasting Insulin (post-intervention) } \\
\hline Vizza 2016 & 20 & 12 & 7 & 10 & 6 & 6 & $1.8 \%$ & $10.00[-0.10,20.10]$ & & & 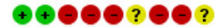 \\
\hline Almenning 2015 & 16.2 & 6.83 & 16 & 18.3 & 11.1 & g & $2.8 \%$ & $-2.10[-10.09,5.89]$ & & & $\odot \odot \odot \odot \odot \odot \odot \odot ?$ \\
\hline Saremi 2013 & 12 & 6.18 & 11 & 14.92 & 9.49 & 11 & $3.8 \%$ & $-2.92[-9.61,3.77]$ & & - & $+?$ ? ? ? \\
\hline Sa 2015 & 5.33 & 3.47 & 14 & 11.3 & 6.05 & 13 & $10.0 \%$ & $-5.97[-9.73,-2.21]$ & $\longrightarrow$ & & $\odot \odot \odot ? \theta$ \\
\hline Stener-Victorin 2009 & 6.4 & 2.8 & 5 & 7.8 & 3.1 & 6 & $11.1 \%$ & $-1.40[-4.89,2.09]$ & $\ldots$ & & \\
\hline Turan 2015 & 13.9 & 3.7417 & 14 & 14.5 & 3.2 & 16 & $16.9 \%$ & $-0.60[-3.11,1.91]$ & $\rightarrow$ & & $\odot \odot \odot \odot \odot ? \odot \odot ?$ \\
\hline Saremi 2016 & 11.46 & 2.2 & 10 & 14.1 & 1.2 & 10 & $25.8 \%$ & $-2.64[-4.19,-1.09]$ & - & & ? †อ厂? ? ? ? ? \\
\hline Vigorito 2007 & 18.3 & 3 & 45 & 20.4 & 3.6 & 45 & $27.9 \%$ & $-2.10[-3.47,-0.73]$ & $=$ & & ? ? $\odot \odot ?$ ? ?๑९ \\
\hline Subtotal $(95 \% \mathrm{Cl})$ & & & 122 & & & 116 & $100.0 \%$ & $-2.11[-3.49,-0.73]$ & $\bullet$ & & \\
\hline \multicolumn{12}{|c|}{$\begin{array}{l}\text { Heterogeneity: Tau }{ }^{2}=1.29 ; \mathrm{Chi}^{2}=11.60, \mathrm{df}=7(\mathrm{P}=0.11) ; \mathrm{F}^{2}=40 \% \\
\text { Test for overall effect: } Z=3.00(\mathrm{P}=0.003)\end{array}$} \\
\hline & & & & & & & & & $-20 \quad-10$ & $\begin{array}{lll}1 & 1 & 1 \\
0 & 10 & 20\end{array}$ & \\
\hline \multicolumn{12}{|c|}{ Test for subgroup differences: $\mathrm{Chi}^{2}=0.08, \mathrm{df}=1(\mathrm{P}=0.77), \mathrm{I}^{2}=0 \%$} \\
\hline \multirow{2}{*}{\multicolumn{12}{|c|}{ Risk of bias legend }} \\
\hline \multirow{2}{*}{\multicolumn{12}{|c|}{$\begin{array}{l}\text { (A) Random sequence generation (selection bias) } \\
\text { (B) Allocation concealment (selection bias) }\end{array}$}} \\
\hline & & & & & & & & & & & \\
\hline \multirow{2}{*}{\multicolumn{12}{|c|}{$\begin{array}{l}\text { (C) Blinding of participants and personnel (performance bias) } \\
\text { (D) Blinding of outcome assessment (detection bias) }\end{array}$}} \\
\hline \multirow{2}{*}{\multicolumn{12}{|c|}{ (E) Incomplete outcome data (attrition bias) }} \\
\hline & & & & & & & & & & & \\
\hline \multicolumn{12}{|c|}{ (F) Selective reporting (reporting bias) } \\
\hline \multicolumn{12}{|c|}{$\begin{array}{l}\text { (G) Group similarity at baseline (other bias) } \\
\text { (H) Adherence (other bias) }\end{array}$} \\
\hline $\begin{array}{l}\text { (H) Adherence (other } \\
\text { (I) Contamination (oth }\end{array}$ & $\begin{array}{l}\text { ias) } \\
\text { er bias) }\end{array}$ & & & & & & & & & & \\
\hline q. 3 Forest plo & of compari & son: exerc & cise vs & s. control ol & utcome: $f_{c}$ & asting & g insuli & in plasma levels (ulU/r & $\mathrm{mL})$ & & \\
\hline
\end{tabular}


results of the removed trial may have varied due to the mode of exercise used (resistance training) or the use of a placebo.

A statistical effect of exercise versus control on FI was shown in multiple subgroups (Additional file 1: Table S5). We found a change in FI from baseline to post-intervention in studies with participants who were overweight (BMI $25-29.9 \mathrm{~kg} / \mathrm{m}^{2}, \mathrm{MD}-3.25 \mu \mathrm{IU} / \mathrm{mL}$, $95 \%$ CI -5.27 to -1.22 ; 5 trials, 168 participants, $I^{2}=$ $75 \%)$; interventions that were aerobic exercise-based (MD $-2.22 \mu \mathrm{IU} / \mathrm{mL}, 95 \% \mathrm{CI}-3.57$ to -0.86 ; 6 trials, 192 participants, $I^{2}=10 \%$ ); $\leq 12$ weeks duration (MD $2.92 \mu \mathrm{IU} / \mathrm{mL}, 95 \% \mathrm{CI}-4.91$ to -0.93 ; 7 trials, 225 participants, $I^{2}=93 \%$ ); and supervised and combined supervised and unsupervised (MD - $2.54 \mu \mathrm{IU} / \mathrm{mL}, 95 \% \mathrm{CI}-$ 4.82 to -0.26 ; 6 trials, 214 participants, $I^{2}=94 \%$, and $\mathrm{MD}-3.08 \mu \mathrm{IU} / \mathrm{mL}, 95 \% \mathrm{CI}-5.63$ to $-0.53 ; 2$ trials, 38 participants, $I^{2}=17 \%$, respectively).

Compared with control, favourable effects of exercise on FI post-intervention values were found for participants who were overweight (MD - $2.27 \mu \mathrm{IU} / \mathrm{mL}, 95 \% \mathrm{CI}-3.24$ to $-1.31 ; 5$ trials, 168 participants, $I^{2}=0 \%$ ); interventions that were aerobic exercise-based $(\mathrm{MD}-2.48 \mu \mathrm{IU} / \mathrm{mL}$, $95 \%$ CI -3.92 to $-1.04 ; 5$ trials, 167 participants, $I^{2}=$ $10 \%$ ); $\leq 12$ weeks duration (MD - $1.80 \mu \mathrm{IU} / \mathrm{mL}, 95 \% \mathrm{CI}-$ 3.18 to -0.42 ; 6 trials, 200 participants, $I^{2}=32 \%$ ); and supervised (MD $-2.39 \mu \mathrm{IU} / \mathrm{mL}, 95 \% \mathrm{CI}-3.62$ to $-1.17 ; 5$ trials, 189 participants, $\left.I^{2}=30 \%\right)$.

\section{HOMA-IR}

Greater reductions in HOMA-IR change scores were evident for exercise versus control (MD - 0.57, 95\% CI - 0.99 to -0.14 ; 8 trials, 173 participants, $I^{2}=87 \%$; Table 3; Fig. 4), but the comparison of post-intervention HOMA-IR values did not reveal a significant exercise effect. In a sensitivity analysis including only trials at a low risk of bias, the effect of exercise was maintained (MD $-0.81,95 \% \mathrm{CI}-1.40$ to 0.21 ; 97 participants, 4 trials, $I^{2}=77 \%$ ) for HOMA-IR changes. Only one trial had a sample size of $\geq 30$ participants [117], so a corresponding sensitivity analysis was not possible. We rated the result as very low-quality evidence due to unclear or high risk of selection, detection, attrition, and reporting bias, contamination, low adherence, considerable heterogeneity with minimal or no overlap of confidence intervals, small number of participants, and a null or negligible effect and appreciable benefit included in the confidence interval for the mean difference (Table 4).

In the investigation of heterogeneity, removing the most extreme value [32] had a negligible effect on the $I^{2}$ (89\%), but a small effect was maintained (MD - 0.50, $95 \%$ CI -0.96 to -0.05$)$. Similarly, the $I^{2}$ statistic was still representative of at least substantial heterogeneity in sub-analyses. The lowest reported value $\left(I^{2}=60 \%\right)$ was in the aerobic exercise intervention subgroup.
Subgroup analyses revealed statistical effects on HOMA-IR change from baseline for aerobic exercise interventions (MD $-0.73,95 \% \mathrm{CI}-1.24$ to $-0.21 ; 5$ trials, 102 participants, $I^{2}=60 \%$ ); $\leq 12$ weeks duration (MD $0.69,95 \% \mathrm{CI}-1.13$ to -0.26 ; 6 trials, 135 participants, $I^{2}=89 \%$ ); and supervised delivery (MD - 0.80, 95\% CI 1.19 to -0.42 ; 5 trials, 124 participants, $I^{2}=76 \%$ ); and for participants in the overweight subgroup (MD -0.83 , $95 \%$ CI -1.39 to -0.26 ; 4 trials, 78 participants, $I^{2}=$ $75 \%)$. Post-intervention subgroup analysis revealed no effects (Additional file 1: Table S5).

\section{Circulating lipids}

Seven trials (225 participants) were included in the analysis of all lipid-related outcomes (TC, LDL-C, and HDL-C, and triglycerides; Fig. 5; Table 3). A statistically significant effect of exercise versus control was observed for TC change scores (MD - $5.88 \mathrm{mg} / \mathrm{dL}, 95 \% \mathrm{CI}-9.92$ to $-1.83 ; I^{2}=35 \%$ ), LDL-C (MD $-7.39 \mathrm{mg} / \mathrm{dL}, 95 \% \mathrm{CI}$ -9.83 to $-4.95 ; I^{2}=0 \%$ ), and triglycerides (MD -4.78 $\mathrm{mg} / \mathrm{dL}, 95 \% \mathrm{CI}-7.52$ to $-2.05 ; I^{2}=3 \%$ ), but not for HDL-C (Table 3). Post-intervention values analysis of lipid-related outcomes revealed an effect on TC (MD $6.35 \mathrm{mg} / \mathrm{dL}, 95 \% \mathrm{CI}-10.76$ to $\left.-1.95 ; I^{2}=0 \%\right)$ and LDL-C (MD $-6.68 \mathrm{mg} / \mathrm{dL}, 95 \% \mathrm{CI}-11.66$ to -1.70 ; $I^{2}=0 \%$ ) (Table 3 ). We rated these results as low-quality evidence (Table 4) due to high or unclear risk of selection bias, detection bias, reporting bias, contamination, and imprecision due to small number of participants and wide confidence intervals in the included trials.

In sensitivity analyses, the favourable effects of exercise versus control on TC, LDL-C, and triglycerides change scores were maintained in studies with a low risk of bias (MD $-5.94 \mathrm{md} / \mathrm{dL}, 95 \% \mathrm{CI}-10.32$ to $-1.55 ; 5$ trials, 187 participants, $I^{2}=40 \%$; MD $-6.60 \mathrm{mg} / \mathrm{dL}, 95 \%$ CI -9.88 to -3.32 ; 5 trials, 187 participants, $I^{2}=14 \%$; and $\mathrm{MD}-5.97 \mathrm{mg} / \mathrm{dL}, 95 \% \mathrm{CI}-10.91$ to -1.03 ; 5 trials, 187 participants, $I^{2}=33 \%$, respectively), and in larger trials $(\mathrm{MD}-3.74 \mathrm{mg} / \mathrm{dL}, 95 \% \mathrm{CI}-6.13$ to -1.35 ; 120 participants, 2 trials, $I^{2}=0 \%$; MD -8.58 , $95 \% \mathrm{CI}-11.44$ to - 5.71; 120 participants, 2 trials, $I^{2}=0 \%$; and MD -3.62 $\mathrm{mg} / \mathrm{dL}, 95 \% \mathrm{CI}-6.22$ to $-1.02 ; 120$ participants, 2 trials, $I^{2}=0 \%$, respectively). Sensitivity analyses for LDL-C post-intervention values showed a retained effect when trials with a high risk of bias were excluded (MD - 8.64 $\mathrm{mg} / \mathrm{dL}, 95 \% \mathrm{CI}-16.30$ to -0.98 ; 5 trials, 187 participants, $I^{2}=22 \%$ ), but not when smaller trials were removed.

Subgroup analyses of TC change (Additional file 1: Table S6) revealed statistical effects for interventions that were $\leq 12$ weeks duration $(\mathrm{MD}-5.94 \mathrm{mg} / \mathrm{dL}, 95 \% \mathrm{CI}-$ 10.32 to -1.55 ; 5 trials, 187 participants, $\left.I^{2}=37 \%\right)$ or supervised (MD $-7.25 \mathrm{mg} / \mathrm{dL}, 95 \% \mathrm{CI}-11.92$ to $-2.58 ; 5$ trials, 189 participants, $\left.I^{2}=48 \%\right)$. There was also an effect 


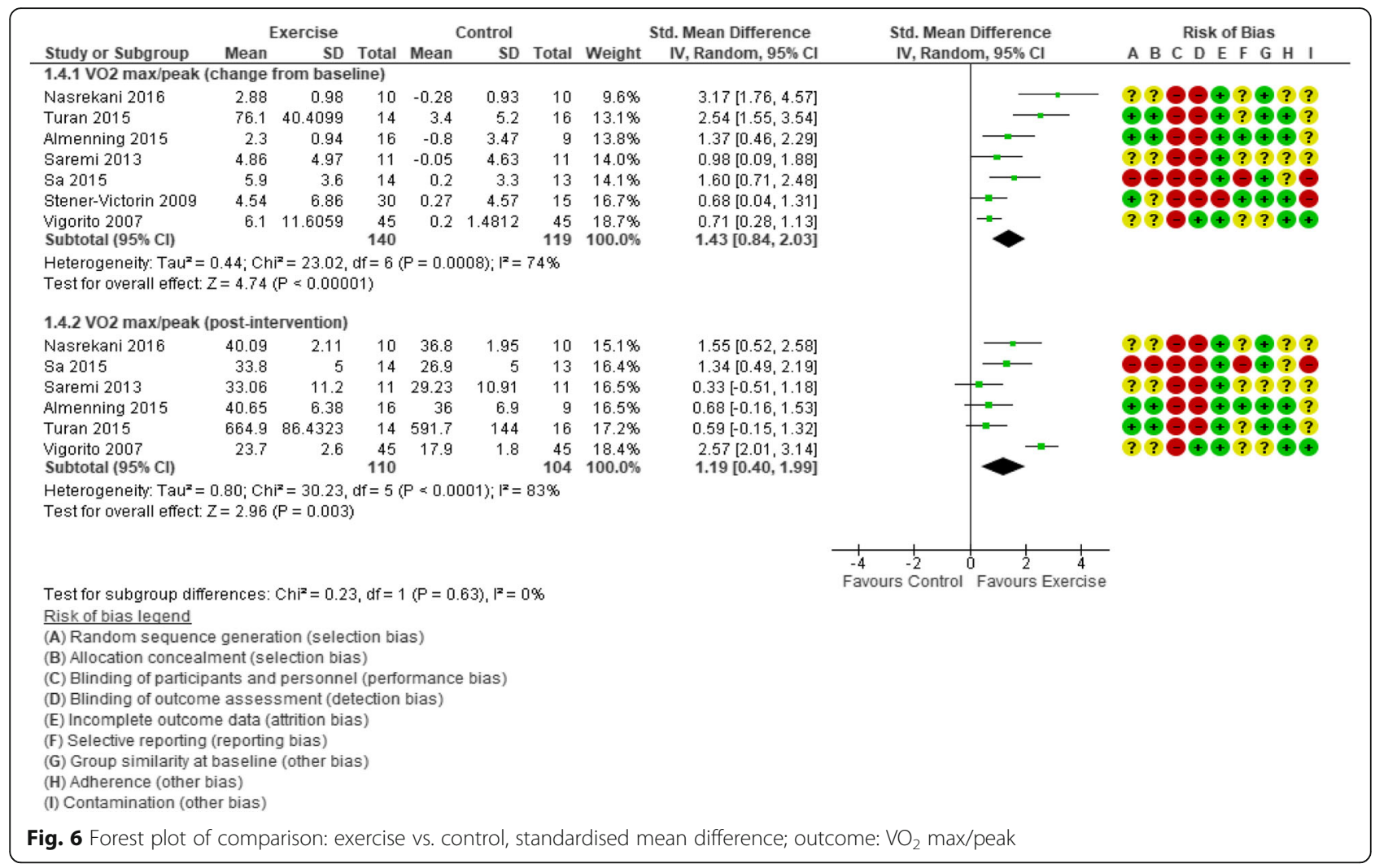

in subgroup analysis for change from baseline (MD - 6.68 $\mathrm{mg} / \mathrm{dL}, 95 \% \mathrm{CI}-13.00$ to -0.35 ; 5 trials, 167 participants, $I^{2}=39 \%$ ) and post-intervention TC values (MD - 6.90 $\mathrm{mg} / \mathrm{dL}, 95 \% \mathrm{CI}-11.90$ to $-1.90 ; 5$ trials, 167 participants, $\left.I^{2}=0 \%\right)$ in aerobic exercise interventions. Subgroup analysis of post-intervention TC also revealed an effect when interventions > 12 weeks (MD $-9.92 \mathrm{mg} / \mathrm{dL}, 95 \% \mathrm{CI}-$ 17.81 to $-2.04 ; 2$ trials, 38 participants, $I^{2}=0 \%$ ) or were supervised (MD - $6.76 \mathrm{mg} / \mathrm{dL}, 95 \% \mathrm{CI}-11.27$ to $-2.26 ; 5$ trials, 189 participants, $\left.I^{2}=0 \%\right)$.

In subgroup analyses for LDL-C change from baseline, a statistically favourable exercise effect was found in trials consisting of interventions $\leq 12$ weeks duration (MD $-6.60 \mathrm{mg}$ / dL, $95 \% \mathrm{CI}-9.88$ to -3.32 ; 5 trials, 187 participants, $I^{2}=$ $13 \%$ ) or supervised (MD $-6.70 \mathrm{mg} / \mathrm{dL}, 95 \% \mathrm{CI}-10.29$ to 3.12; 5 trials, 189 participants, $I^{2}=23 \%$ ). Subgroup analysis for LDL-C post-intervention values revealed statistical effects in participants with BMI of $25-29.9 \mathrm{~kg} / \mathrm{m}^{2}$ (MD $-9.54 \mathrm{mg}$ / dL, $95 \% \mathrm{CI}-18.71$ to -0.36 ; 5 trials, 168 participants, $I^{2}=$ $22 \%$ ), and interventions of $\leq 12$ weeks duration (MD -8.64 $\mathrm{mg} / \mathrm{dL}, 95 \% \mathrm{CI}-16.30$ to -0.98 ; 5 trials, 187 participants, $I^{2}=22 \%$ ), supervised (MD $-7.58 \mathrm{mg} / \mathrm{dL}, 95 \% \mathrm{CI}-13.73$ to - 1.43; 5 trials, 187 participants, $I^{2}=24 \%$ ), or aerobic (MD: $-5.87 \mathrm{mg} / \mathrm{dL}, 95 \% \mathrm{CI}-11.68$ to $-0.07 ; 5$ trials, 167 participants, $I^{2}=0 \%$; Additional file 1: Table S6).

For HDL-C, only subgroup analyses of resistance training interventions showed a negative effect on change from baseline scores (MD $-2.19 \mathrm{mg} / \mathrm{dL}, 95 \% \mathrm{CI}$ -4.21 to -0.18 ; 2 trials, 37 participants, $I^{2}=0 \%$ ) and a positive effect on post-intervention values (MD $7.29 \mathrm{mg} /$ dL, 95\% CI 1.11 to 13.46 ; 2 trials, 37 participants, $I^{2}=$ 17\%; Additional file 1: Table S6). No effects of exercise were found in other HDL-C subgroup analyses.

Compared with control, exercise had a favourable effect on triglyceride values in the following subgroups: BMI 25-29.9 kg/m² (MD - 8.17 mg/dL, 95\% CI - 14.44 to -1.89 ; 5 trials, 167 participants, $I^{2}=13 \%$ ); aerobic exercise interventions (MD - $6.80 \mathrm{mg} / \mathrm{dL}, 95 \% \mathrm{CI}-13.12$ to -0.48 ; 5 trials, 167 participants, $I^{2}=5 \%$ ); $\leq 12$ weeks duration (MD $-6.06 \mathrm{mg} / \mathrm{dL}, 95 \% \mathrm{CI}-10.82$ to $-1.31 ; 5$ trials, 187 participants, $\left.I^{2}=30 \%\right)$; and supervised interventions (MD $-5.91 \mathrm{mg} / \mathrm{dL}, 95 \% \mathrm{CI}-10.75$ to $-1.06 ; 5$ trials, 189 participants, $I^{2}=29 \%$; Additional file 1: Table S6). Analysis of triglyceride post-intervention values revealed an effect of exercise in trials $>12$ weeks only (MD - $13.85 \mathrm{mg} / \mathrm{dL}, 95 \% \mathrm{CI}-26.33$ to -1.36 ; 2 trials, 38 participants, $\left.I^{2}=0 \%\right)$.

\section{Secondary outcomes}

\section{Maximal or peak oxygen uptake}

A large statistical effect of exercise versus control was found for both change from baseline and post-intervention $\mathrm{VO}_{2}$ max/peak values (SMD 1.43, 95\% CI 0.84 to 2.03; 259 participants, 7 trials, $I^{2}=74 \%$, and SMD $1.19,95 \%$ CI 0.40 
to $1.99 ; I^{2}=83 \%$, respectively; Fig. 6 ). With the inclusion of only studies that reported relative $\mathrm{VO}_{2} \max$ /peak values (i.e., expressed as $\mathrm{ml} / \mathrm{kg} / \mathrm{min}$ ), the effect of exercise was maintained in both change scores and post-intervention values (MD $3.84 \mathrm{ml} / \mathrm{kg} / \mathrm{min}, 95 \% \mathrm{CI} 2.87$ to $4.81 ; 6$ trials, 229 participants, $I^{2}=17 \%$, and MD $5.01 \mathrm{ml} / \mathrm{kg} / \mathrm{min}, 95 \%$ CI 3.48 to $6.54 ; 5$ trials, 184 participants, $I^{2}=42 \%$, respectively).

For the $\mathrm{SMD} \mathrm{VO}_{2}$ max/peak change sensitivity analysis, an effect remained when small trials (SMD 1.21, 95\% CI 0.29 to 2.12 ; 3 trials, 165 participants, $I^{2}=83 \%$ ) and those with a high risk of bias (SMD 1.63, 95\% CI 0.78 to 2.48; 5 trials, 187 participants, $I^{2}=80 \%$ ) were removed. SMD was also used to complete post-intervention sensitivity analysis for sample size; 2 trials (120 participants) $[116,117]$ were included, but the effect was lost. However, it remained when trials with a high risk of bias were removed (SMD 1.16, 95\% CI 0.21 to 2.12; 5 trials, 187 participants, $I^{2}=87 \%$ ).

When we considered only relative $\mathrm{VO}_{2}$ max/peak change scores, the effect of exercise was maintained when removing small studies (MD $1.21 \mathrm{ml} / \mathrm{kg} / \mathrm{min}$, 95\% CI 0.29 to $2.12,165$ participants, 3 trials, $I^{2}=83 \%$ ) and studies with a high risk of bias (MD $3.35 \mathrm{ml} / \mathrm{kg} / \mathrm{min}$, 95\% CI 2.59 to 4.10 ; 157 participants, 4 trials, $I^{2}=0 \%$ ). All trials in the post-intervention relative $\mathrm{VO}_{2}$ max/peak analysis were considered low risk of bias, so this sensitivity analysis was not possible.

For ease of interpretation, we performed subgroup analyses on the relative $\mathrm{VO}_{2}$ max/peak data. Subgroup analysis of the change from baseline relative $\mathrm{VO}_{2}$ max/peak values revealed statistical improvements with aerobic exercise, any intervention duration, and for participants with a BMI of $25-29.9 \mathrm{~kg} / \mathrm{m}^{2}$. The post-intervention pooled analysis showed an effect of exercise on relative $\mathrm{VO}_{2} \max /$ peak in four subgroups: participants with a BMI of 25$29.9 \mathrm{~kg} / \mathrm{m}^{2}$, aerobic exercise interventions, $\leq 12$ weeks, and supervised (Additional file 1: Table S7).

In one trial reporting data from a post-intervention 16-week follow-up [111], a $12 \%$ increase in $\mathrm{VO}_{2} \max$ $(4.11 \pm 5.20 \mathrm{ml} / \mathrm{kg} / \mathrm{min} ; p=.001)$ from baseline was still evident in the exercise group. The corresponding change for control (7\%) was not statistically significant, and there were no significant differences between groups.

\section{Resting heart rate}

A pooled analysis of four trials (156 participants) indicated no effect of exercise on the change scores of resting heart rate (RHR) values (Table 3). However, in these trials, RHR post-intervention values were statistically lower in the exercise interventions versus control (MD -3.26 beats $/ \mathrm{min}, 95 \%$ CI -4.93 to $-1.59 ; I^{2}=0 \%$ ). When post-intervention sensitivity analyses were completed, this effect was still evident in larger trials (MD 3.18 beats $/ \mathrm{min}, 95 \% \mathrm{CI}-5.59$ to $-0.77,3$ trials, 145 participants, $I^{2}=16 \%$ ) and those with a low risk of bias (MD -3.53 beats/min, $95 \%$ CI -5.28 to $-1.78 ; 2$ trials, 120 participants, $I^{2}=0 \%$ ).

In subgroup analyses, there were statistical effects of exercise compared with control on both RHR change from baseline and post-intervention values in interventions that were aerobic exercise-based (Additional file 2: Figure S3), and those that were supervised. Post-intervention subgroup analysis also revealed effects in interventions of $\leq 12$ weeks and when participants had a BMI $25-29.9 \mathrm{~kg} / \mathrm{m}^{2}$ at study entry (Additional file 1 : Table S7).

\section{Body mass and body mass index}

We found a statistical effect of exercise on BMI post-intervention values (MD $-1.02 \mathrm{~kg} / \mathrm{m}^{2}, 95 \% \mathrm{CI}-$ 1.81 to -0.23 ; 10 trials, 272 participants, $I^{2}=0 \%$ ) compared with control (Table 3). When trials with a high risk of bias were removed from the sensitivity analysis for BMI post-intervention values, an effect remained (MD $-0.95 \mathrm{~kg} / \mathrm{m}^{2}, 95 \% \mathrm{CI}-1.78$ to $-0.12 ; 6$ trials, 207 participants, $I^{2}=0 \%$ ), but not when small trials were removed.

Subgroup analysis revealed a statistical reduction in BMI change scores with exercise in studies consisting of participants with BMI $\geq 30 \mathrm{~kg} / \mathrm{m}^{2}$. Analysis of BMI change from baseline also revealed a statistical decrease with aerobic exercise interventions, but a statistical increase with resistance training interventions (Additional file 1: Table S7).

Post-intervention subgroup analysis revealed statistical reductions in BMI with interventions that were aerobic exercise-based, supervised, and $\leq 12$ weeks, and also in participants with a BMI of $25-29.9 \mathrm{~kg} / \mathrm{m}^{2}$ (Additional file 1: Table S7).

The meta-analysis revealed no effect of exercise versus control on body mass change from baseline or post-intervention values (Table 3). However, we found statistical effects of exercise versus control on change in body mass from baseline to post-intervention for studies involving participants with $\mathrm{BMI} \geq 30 \mathrm{~kg} / \mathrm{m}^{2}$. No other subgroup analyses indicated such an effect (Additional file 1: Table S7).

Follow-up reporting (16-weeks post-intervention) of BMI from one trial [111] showed no statistically significant within-group changes or between-group differences in either exercise or control arms. The authors [111] also reported similar findings immediately post-intervention.

\section{Waist and hip circumference and waist-to-hip ratio}

Analysis of waist circumference (WC) change scores, but not post-intervention values, revealed a statistically significant beneficial effect of exercise compared with controls $(\mathrm{MD}-2.62 \mathrm{~cm}, 95 \% \mathrm{CI}-4.13$ to -1.11 ; 7 trials, 
221 participants, $I^{2}=53 \%$; Table 3 ). The favourable effect of exercise on WC change remained when trials with a low risk of bias (MD $-1.51 \mathrm{~cm}, 95 \% \mathrm{CI}-2.26$ to - 0.76; 167 participants, 4 trials, $I^{2}=0 \%$ ) and larger sample sizes (MD $-1.48 \mathrm{~cm}, 95 \% \mathrm{CI}-2.26$ to $-0.71 ; 120$ participants, 2 trials, $\left.I^{2}=0 \%\right)$ were analysed separately. When the largest outlier [108] was removed from this analysis, the $I^{2}$ was reduced to $0 \%$ and an effect remained ( $\mathrm{MD}-1.68 \mathrm{~cm}, 95 \% \mathrm{CI}-2.38$ to -0.99 ).

In subgroup analyses for WC change, exercise had a statistical effect in studies with participants with BMI of $25-29.9 \mathrm{~kg} / \mathrm{m}^{2}$ and $\geq 30 \mathrm{~kg} / \mathrm{m}^{2}, \leq 12$ week's duration, aerobic and resistance-based interventions, and supervised exercise (Additional file 1: Table S7). Subgroup analysis revealed that post-intervention $\mathrm{WC}$ values were statistically lower in exercise interventions with participants with BMI $25-29.9 \mathrm{~kg} / \mathrm{m}^{2}$, aerobic exercise, and supervised exercise (Additional file 1: Table S7).

Data from two trials $[111,118]$ were pooled in the analysis of waist-to-hip ratio (WHR); there was no effect in either change from baseline or post-intervention values analyses.

\section{Body composition}

The pooled MD for body fat percentage change from baseline was statistically significant (MD - 1.39\%, 95\% CI -2.61 to -0.18 ; 3 trials, 60 participants, $I^{2}=30 \%$ ), but not for post-intervention values (Table 3 ). When trials deemed to have a high risk of bias were removed, this statistical effect disappeared. Sensitivity analysis by study size could not be performed for the exercise effect on body fat percentage due to a lack of sufficiently large studies. Moreover, we found no effect of exercise versus control on change from baseline or post-intervention analyses for fat mass and fat-free mass (Table 3).

A statistical effect was found for exercise on body fat percentage change in interventions $\leq 12$ weeks, but this analysis included the same trials as the main analysis. No other statistical effects were found across any of the other subgroup analyses on body fat percentage change (Additional file 1: Table S7). However, body fat percentage was statistically lower post-intervention in exercise interventions that included participants with BMI of 25$29.9 \mathrm{~kg} / \mathrm{m}^{2}$, and aerobic exercise (Additional file 1: Table S7). No effect was evident in the subgroup analysis for fat mass or fat-free mass.

\section{Androgenic, hormonal, and inflammatory markers}

In pooled analyses of change from baseline or post-intervention values, exercise had no beneficial effect on any of the androgenic/hormonal and inflammatory biomarkers/variables [i.e., testosterone, free testosterone, free androgen index (FAI), sex hormone binding globulin (SHBG), Ferriman-Gallwey scores, oestradiol, luteinising hormone (LH), follicle stimulating hormone (FSH), LH/ FSH ratio, progesterone, prolactin, high-sensitivity C-reactive protein, anti-Mullerian hormone $(\mathrm{AMH})$, or adiponectin] when compared with control (Table 3). Similarly, there were no effects in any subgroup analysis for these outcomes (Additional file 1: Table S8).

\section{Psychosocial outcomes}

In two trials (57 participants) that assessed psychosocial outcomes using the PCOS-Q, we found no effect of exercise on any PCOS-Q domain compared with control. Three trials (84 participants) used the SF-36. Data only allowed for change from baseline analysis and no sub-analysis was possible. For SF-36 domains, a favourable effect of exercise versus control was found for physical functioning (MD 11.81, 95\% CI 2.36 to $21.25 ; I^{2}=74 \%$ ), general health (MD 10.05, 95\% CI 3.89 to $16.20 ; I^{2}=0 \%$ ), social functioning (MD 11.75, 95\% CI 2.56 to $20.95 ; I^{2}=$ $6 \%$ ), and mental health (MD 11.70, 95\% CI 1.27 to 22.13; $I^{2}=47 \%$ ) domains (Additional file 2: Figure S5).

There were insufficient data to complete sensitivity analyses; however, all three trials [108, 111, 119] were judged to have a high risk of bias in at least one domain, and only one trial had a sample size $\geq 30$. Heterogeneity was investigated in the physical functioning domain; the largest outlier was removed [108] and the $I^{2}$ was reduced to $33 \%$, whilst an effect was maintained (MD 7.23, 95\% CI 1.66 to 12.80 ). The same trial was removed in the general health analysis, resulting in a reduction in $I^{2}$ to $0 \%$, and a preserved effect (MD 7.97, 95\% CI 1.07 to $4.88)$. When the greatest outliers were removed from the social functioning [119] and mental health [111] domains, both $I^{2}$ values were reduced to $0 \%$, but the effect only remained in the mental health domain (MD 17.84, 95\% CI 7.33 to 28.36 ).

\section{Additional outcomes}

Six trials [32, 108, 111, 117-119] also reported a range of additional outcomes; the key findings from these are presented in Additional file 1: Table S9.

\section{Effects of interventions: Exercise and diet versus control}

Three trials compared exercise and diet combined versus control. Only one of these trials used a control group that was described as no treatment [99]. The other two $[100,107]$ compared exercise, diet and metformin (or placebo) to metformin only groups. As pharmacological intervention was present in each included treatment arm, we assumed that any variation between groups would result from exercise and dietary components.

Due to insufficient data, it was only possible to include two outcomes in the meta-analysis. Meta-analysis of the two trials (68 participants) reporting change from baseline to post-intervention WHR values revealed a small 
but statistically significant effect in favour of exercise and diet (MD $-0.02,95 \% \mathrm{CI}-0.03$ to $-0.01 ; I^{2}=0 \%$; Additional file 2: Figure S6). The effect was not replicated in the post-intervention value analysis.

We found no effect of exercise and diet combined versus control on the change from baseline to postintervention SHBG concentrations (Additional file 2: Figure S7). There were insufficient data to complete analysis of post-intervention values or subgroups. Individual outcomes were also reported by each of these trials, which are summarised in Additional file 1: Table S10.

\section{Effects of interventions: Exercise and diet versus diet}

Three trials had intervention arms that compared the combination of exercise and diet to diet only $[33,98,104]$. Analyses of change from baseline and post-intervention values from these trials revealed no statistical difference between combined exercise and diet or diet only interventions for any assessed primary outcome (FBG, FI, and
HOMA-IR; all very low-quality evidence; Table 5) or secondary outcome (body weight, BMI, WC, body fat, fat-free mass, testosterone, SHBG, and FAI; Additional file 1: Table S11). There were insufficient data to complete subgroup analyses within this comparison.

All three trials reported a range of other outcomes not included in this meta-analysis; these are summarised in Additional file 1: Table S12.

\section{Effects of interventions: Exercise vs diet, and exercise and diet vs exercise}

Only one trial [105] compared exercise with diet, and exercise combined with diet versus exercise only. Effects in the diet only and combined diet and exercise group have been reported above and in Additional file 1: Table S12. The exercise-only intervention reduced BMI $\left(-0.85 \mathrm{~kg} / \mathrm{m}^{2}, 95 \%\right.$ CI -1.69 to $-0.02 ; P<.05)$, but these changes were smaller than those seen in the other treatment arms. Upper body fat was statistically reduced only in the exercise group (-

Table 5 Summary of findings for primary outcomes: exercise and diet versus diet

\begin{tabular}{|c|c|c|c|c|c|c|}
\hline \multicolumn{7}{|c|}{ Exercise and diet compared to Diet for women with PCOS } \\
\hline \multicolumn{7}{|c|}{$\begin{array}{l}\text { Patient or population: women with PCOS } \\
\text { Setting: } \\
\text { Intervention: exercise and diet } \\
\text { Comparison: Diet }\end{array}$} \\
\hline \multirow[t]{2}{*}{ Outcomes } & \multicolumn{2}{|c|}{ Anticipated absolute effects* $(95 \%$ Cl) } & \multirow{2}{*}{$\begin{array}{l}\text { Relative } \\
\text { effect } \\
(95 \% \text { Cl) }\end{array}$} & \multirow{2}{*}{$\begin{array}{l}\text { № of } \\
\text { participants } \\
\text { (studies) }\end{array}$} & \multirow{2}{*}{$\begin{array}{l}\text { Certainty } \\
\text { of the } \\
\text { evidence } \\
\text { (GRADE) }\end{array}$} & \multirow[t]{2}{*}{ Comments } \\
\hline & Risk with Diet & Risk with exercise and diet & & & & \\
\hline $\begin{array}{l}\text { Fasting blood glucose } \\
\text { (change from baseline) } \\
\text { follow-up: range } 16 \\
\text { weeks to } 20 \text { weeks }\end{array}$ & $\begin{array}{l}\text { The mean fasting blood } \\
\text { glucose (change from } \\
\text { baseline) ranged from } \\
-7.0 \text { to }-3.2 \mathrm{mg} / \mathrm{dL}\end{array}$ & $\begin{array}{l}\text { The mean fasting blood glucose } \\
\text { (change from baseline) in the } \\
\text { intervention group was } 2.92 \mathrm{mg} / \mathrm{dL} \\
\text { higher ( } 0.4 \text { lower to } 6.23 \text { higher) }\end{array}$ & - & 78 (2 RCTs) & $\begin{array}{l}\text { ĐOOO } \\
\text { VERY } \\
\text { LOW } a, b\end{array}$ & $\begin{array}{l}\text { We are uncertain about } \\
\text { the effect of exercise and } \\
\text { diet on fasting blood } \\
\text { glucose (change from } \\
\text { baseline). }\end{array}$ \\
\hline $\begin{array}{l}\text { Fasting insulin (change } \\
\text { from baseline) follow- } \\
\text { up: range } 12 \text { weeks to } \\
20 \text { weeks }\end{array}$ & $\begin{array}{l}\text { The mean fasting } \\
\text { insulin (change from } \\
\text { baseline) ranged from } \\
-2.9 \text { to }-18.54 \mu \mathrm{U} / \mathrm{ml}\end{array}$ & $\begin{array}{l}\text { The mean fasting insulin (change } \\
\text { from baseline) in the intervention } \\
\text { group was } 2.22 \mu \mathrm{U} / \mathrm{ml} \text { higher ( } 3.7 \\
\text { lower to } 8.14 \text { higher) }\end{array}$ & - & 90 (3 RCTs) & $\begin{array}{l}\oplus O O O \\
\text { VERY } \\
\text { LOW }{ }^{a, c, d}\end{array}$ & $\begin{array}{l}\text { We are uncertain about } \\
\text { the effect of exercise and } \\
\text { diet on fasting insulin } \\
\text { (change from baseline). }\end{array}$ \\
\hline $\begin{array}{l}\text { HOMA-IR (change from } \\
\text { baseline) follow-up: } \\
\text { range } 16 \text { weeks to } 20 \\
\text { weeks }\end{array}$ & $\begin{array}{l}\text { The mean HOMA-IR } \\
\text { (change from baseline) } \\
\text { ranged from }-0.74 \text { to } \\
-0.56\end{array}$ & $\begin{array}{l}\text { The mean HOMA-IR (change from } \\
\text { baseline) in the intervention group } \\
\text { was } 0.01 \text { lower ( } 0.45 \text { lower to } 0.43 \\
\text { higher) }\end{array}$ & - & 78 (2 RCTs) & $\begin{array}{l}\text { ĐOOO } \\
\text { VERY } \\
\text { LOW }^{a, b}\end{array}$ & $\begin{array}{l}\text { We are uncertain about } \\
\text { the effect of exercise and } \\
\text { diet on HOMA-IR (change } \\
\text { from baseline). }\end{array}$ \\
\hline
\end{tabular}

*The risk in the intervention group (and its 95\% confidence interval) is based on the assumed risk in the comparison group and the relative effect of the intervention (and its 95\% Cl). Cl: Confidence interval; MD: Mean difference

GRADE Working Group grades of evidence

High certainty: We are very confident that the true effect lies close to that of the estimate of the effect

Moderate certainty: We are moderately confident in the effect estimate: The true effect is likely to be close to the estimate of the effect, but there is a possibility that it is substantially different

Low certainty: Our confidence in the effect estimate is limited: The true effect may be substantially different from the estimate of the effect Very low certainty: We have very little confidence in the effect estimate: The true effect is likely to be substantially different from the estimate of effect

\footnotetext{
Explanations

${ }^{a}$ All trials were at an unclear risk of selection bias, reporting bias, contamination, and adherence issues. All trials were at a high risk of detection bias and attrition bias. Therefore, we downgraded by one level

${ }^{\mathrm{b}}$ Small number of participants, only two trials, and wide confidence intervals in the included trials. Therefore, we downgraded by two levels

'Substantial heterogeneity was observed. Therefore, we downgraded by one level

${ }^{\mathrm{d}}$ Small number of participants and trials, wide confidence intervals, and null/negligible effect and appreciable benefit included in the confidence interval for the mean difference. Therefore, we downgraded by two levels
} 
$1.57 \mathrm{~kg}, 95 \% \mathrm{CI}-2.86$ to $-0.28 ; P<.05)$ and mean follicle number exhibited the greatest improvement in the exercise-only group $(P<.01)$. No within-group effects were reported for body fat $(\%)$, lower body fat $(\mathrm{kg})$, lean body mass, free testosterone, insulin-like growth factor-1, insulin-like growth factor binding protein-1, FBG, FI, HOMA-IR, LH, FSH, testosterone, SHBG, T/SHBG ratio, $\mathrm{AMH}$, or mean ovarian volume.

\section{Discussion}

\section{Summary of the main results}

Our systematic review provides up-to-date evidence supporting the incorporation of exercise interventions in the management of PCOS. When exercise was compared with control, we noted statistically beneficial changes from baseline to post-intervention and more favourable post-intervention values for $\mathrm{FI}, \mathrm{TC}$, LDL-C, and $\mathrm{VO}_{2}$ max. Statistically positive change from baseline scores was also observed for HOMA-IR, triglycerides, WC, and body fat percentage, whereas, statistically lower post-intervention values were additionally found for BMI and RHR. In an analysis of a limited number of studies, compared with control, a small statistical effect in favour of exercise and diet was evident for WHR, but not for SHBG. In the exercise and diet versus diet only comparison, we found no evidence of effect in any outcome; however, there were strikingly scant data available (Additional files 3 and Additional file 4).

\section{Primary outcomes}

We found a small change in SBP from baseline to post-intervention with supervised exercise versus control. To our knowledge, this is the first systematic review to report on the effects of exercise on blood pressure in women with PCOS. Existing evidence from the general population suggests that aerobic exercise interventions induce the greatest improvements to SBP and DBP in hypertensive participants [124], with less marked effects in normotensive participants (small decreases in DBP and no effect on SBP). The mean SBP $(116 \mathrm{mmHg})$ and DBP $(73 \mathrm{mmHg})$ values in our review indicates that most PCOS participants were normotensive at baseline; thus, a large effect was not anticipated.

Regarding surrogate markers of IR, we found a statistically beneficial change (FI and HOMA-IR) and more favourable post-intervention values (FI) with exercise compared with control. Subgroup analyses also indicate that the greatest improvements are noted in participants who were overweight or obese and from shorter duration, supervised aerobic-based interventions. These findings agree with those of two previous systematic reviews, which however, did not make the distinction between exercise, diet or their combination, but instead compared lifestyle interventions to control [125, 126].
The more recent of these reviews [125] reported a small, but statistically significant effect on FI change (MD $2.1 \mu \mathrm{IU} / \mathrm{mL}, 95 \% \mathrm{CI}-3.3$ to $-1.0 ; 5$ trials, $\left.I^{2}=0 \%\right)$. The other review [126] also compared the effect of lifestyle to a minimal treatment intervention on FI showing a statistical effect on FI post-intervention values favouring lifestyle (MD $-2.02 \mu \mathrm{IU} / \mathrm{mL}, 95 \% \mathrm{CI}-3.28$ to -0.77 ; 144 participants, 5 trials, $\left.I^{2}=0 \%\right)$. Herein, we expanded on these previous findings by incorporating a greater number of trials and by separating exercise-only trials, thus revealing that based on the available data the exercise alone effects are comparable to that of lifestyle interventions.

Although the PCOS diagnostic criteria do not currently include IR, it is widely acknowledged that IR plays a key role in the pathophysiology of PCOS [127]. Approximately $50-70 \%$ of women with PCOS have IR and hyperinsulinaemia [128], whereas many also present evidence of glucose intolerance [20]. Hyperinsulinaemia in PCOS further promotes secretion of androgens from the ovarian theca cells, whilst supressing SHBG hepatic secretion, thus increasing free androgens and exacerbating the associated symptoms [129]. Despite the integral role of IR in PCOS, there are scant FI reference values in the literature [130]. One study [131] reported FI levels ranging from 2 to $60 \mu \mathrm{IU} / \mathrm{mL}$ in healthy women $(n=111)$, with a mean value of $17.6 \pm 5.7 \mu \mathrm{IU} / \mathrm{mL}$ in women aged $25-34$ years $(n=22)$. A large-scale case-control study of women with PCOS $(n=1404)$ reported mean FI levels of $14.3 \pm 1.6 \mu \mathrm{IU} / \mathrm{mL}$, which was significantly higher than healthy controls [132]. The mean baseline FI level of intervention participants in our review was $16.21 \mu \mathrm{IU} /$ $\mathrm{mL}$, and a reduction of $\sim 13 \%$ was reported following exercise. Due to the variability of normative FI values in PCOS, it is unclear whether these exercise-induced reductions are clinically meaningful.

Although FI correlates with IR, several studies, especially in normoglycaemic populations [133, 134], have shown that HOMA-IR (calculated based on FI and FBG values) may be a better estimate of insulin sensitivity [135]. In the present review, the mean baseline HOMA-IR for the intervention group participants was 2.99 , which dropped to 2.43 ( $\mathrm{MD}-0.57$ ) following exercise, with no evidence of reduction in the control groups. A generally adopted HOMA-IR cut-off value for the identification of IR is 2.6 [136]. This suggests that exercise may have a clinically significant effect on IR compared with usual care. Furthermore, we found no effect of exercise on FBG. Participants were within normal FBG at baseline; thus, this combined with the effect on FI indicates that less insulin is needed to maintain normoglycaemia following exercise.

In contrast to previous reviews [126, 137], we report an effect of exercise on lipid profiles. Compared to 
control, there were improvements in exercise-induced changes for TC, LDL-C, and triglycerides. Based on data included in our review, the mean baseline values for TC (233 mg/dL) and LDL-C (142 mg/dL) would be classified as borderline high or even elevated in the presence of concomitant CVD risk factors [138]. Post-intervention values for LDL-C were lower for exercise compared to control, but TC levels were comparable (approximately $229 \mathrm{mg} / \mathrm{dL}$ in both). LDL-C appears to play a pivotal role in atherogenesis, with progressively increasing risk of coronary heart disease (CHD) with increasing LDL plasma levels [139]. Conversely, inverse associations between HDL-C and both atherosclerosis severity and CHD risk have been reported, with HDL-C levels $\geq 60$ $\mathrm{mg} / \mathrm{dL}$ potentially protecting against CHD [140]. HDL-C baseline and post-intervention values within this systematic review were $>60 \mathrm{mg} / \mathrm{dL}$, which may partially explain why no effect of exercise was found. However, where TC and LDL-C are elevated at baseline, a statistical effect is evident following exercise but the magnitude of the changes may not be clinically important [141, 142].

Mean baseline triglyceride concentrations were higher in the exercise group $(+11 \mathrm{mg} / \mathrm{dL})$ compared with control, but both groups were within the normal range $(<$ $150 \mathrm{mg} / \mathrm{dL}$ ). Exercise reduced triglyceride levels, but post-intervention analysis revealed that concentrations were still lower in the control groups. Triglycerides are independent predictors of CVD mortality in women [143]; however, the magnitude of the observed exercise-induced triglyceride reduction, within the reported range, is likely to have little clinical relevance. Future research is required to investigate the independent effect of exercise in women with hypertriglyceridaemia.

\section{Secondary outcomes}

We found a statistically and clinically significant effect for $\mathrm{VO}_{2} \max (>3.5 \mathrm{ml} / \mathrm{kg} / \mathrm{min})$ with exercise compared with control. Subgroup analyses revealed that aerobic exercise, regardless of other variables, improved $\mathrm{VO}_{2}$ max in women with PCOS.

Low CRF, as measured by $\mathrm{VO}_{2}$ max, has been associated with increased risk of chronic disease and all-cause mortality [144, 145]. Reduction in $\mathrm{VO}_{2}$ max occurs physiologically with age, but is also often linked to inactivity. The consequences of reduced CRF include impaired capability to exercise, reduced ability to perform activities of daily living, and a lower overall quality of life [146]. Consequently, improving patient $\mathrm{VO}_{2} \max$ is a goal of many lifestyle interventions yet is often overlooked in PCOS. Studies assessing $\mathrm{VO}_{2}$ max in this patient population are limited; two such studies in overweight [147] and lean [148] women with PCOS reveal markedly lower CRF than healthy controls. The only previous relevant systematic review to report on $\mathrm{VO}_{2}$ max/peak [137] found improvements for both lifestyle (i.e., exercise and diet combined; MD $5.09 \mathrm{ml} / \mathrm{kg} / \mathrm{min}$, 95\% CI 3.13 to 7.05, 3 trials, 137 participants) and exercise (MD $4.86 \mathrm{ml} / \mathrm{kg} / \mathrm{min}, 95 \%$ CI 2.83 to $6.88,2$ trials, 125 participants) interventions compared with usual care. Our analysis of relative $\mathrm{VO}_{2}$ max change pooled data from 92 more participants than the review by Haqq et al. [137], and although our effect was marginally smaller, the agreement between these results suggests that exercise can improve CRF in this population.

We also found reductions in WC and body fat in the exercise groups, suggesting that exercise promotes favourable changes to body composition in women with PCOS. As a measure of central/abdominal obesity, WC is considered a better independent predictor of obesity-related disorders than BMI [149]. This may be attributed to the key role of central adiposity in the development of IR and T2DM, even in those with normal BMI [150]. However, despite statistical significance, the exercise-induced WC changes may be of unclear clinical relevance, since the observed average reduction from baseline was $2.8 \%$ (95\% CI 1.31 to 4.24 ), which is less than the suggested $3-5 \%$ reduction considered as clinically significant [151].

Improvements in anthropometric outcomes were reported by an older systematic review [137], but these were largely based on comparing lifestyle (not exercise alone) with control. Similarly, when compared to control, Moran et al. [126] reported statistical reductions in body weight and abdominal adiposity following lifestyle interventions. In our systematic review, when combined exercise and dietary interventions were compared with diet only, both groups demonstrated favourable changes, but there was no evidence of an effect favouring either intervention for any outcome.

We found no statistical effect of exercise on the androgenic profile of women with PCOS compared with control. Where analyses were possible, we found no effect favouring either diet and exercise combined or diet only. This was further supported by subgroup analyses where the evidence of relevant effects was minimal. Typically, the baseline values of women with PCOS included in this current review were below recommended cut-offs for diagnosing hyperandrogenism; testosterone $>2.5$ $\mathrm{nmol} / \mathrm{L}$ and SHBG $<30 \mathrm{nmol} / \mathrm{L}$ [152], which indicates that they were not markedly hyperandrogenic. Moran et al. [126] reported reduced testosterone levels following lifestyle intervention but found no effect on FAI (100 $x$ total testosterone/SHBG), a more valid marker of hyperandrogenism [121]. A review of exercise-induced changes on the androgenic profile of healthy women who were premenopausal [153] found that exercise acutely increases circulating androgens, but the chronic effects are less clear. A similar meta-analysis [154] 
reported a chronic statistical reduction in concentrations of bioavailable testosterone (MD $-0.18 \mathrm{pg} / \mathrm{mL}, 95 \%$ CI -0.29 to $-0.07 ; 1369$ participants, 9 trials, $I^{2}=0 \%$ ) and increased SHBG (MD $3.93 \mathrm{nmol} / \mathrm{L}, 95 \%$ CI 0.98 to 6.87; 1643 participants, 14 trials, $I^{2}=75 \%$ ) following exercise in healthy women. Collectively, these data suggest that exercise interventions may regulate androgenic profiles, but that the optimal dose is unclear, with potential variation in women with menstrual disruption [155].

Finally, there is increasing recognition of the deleterious effects of PCOS on HRQoL and other psychosocial components. However, only three eligible trials measured these outcomes in the exercise versus control comparison. There was no evidence of effect in any of the PCOS-Q domains, but scores were improved in the physical functioning, general health, social functioning, and mental health domains of the SF-36. Our meta-analysis revealed improvements in these outcomes of $\geq 10 \%$ for exercise compared with control, supporting the notion that exercise in these patients may improve their perception of physical and mental wellbeing.

\section{Overall completeness and applicability of evidence}

We completed a comprehensive and systematic search of relevant electronic databases and the reference lists from included publications and relevant reviews. From this, we identified 16 RCTs, one quasi-RCT, and a randomised crossover trial. We located and meta-analysed data from more trials, made a greater number of comparisons, and included a wider range of outcomes when compared to previous systematic reviews $[25,125,126$, 148]. To our knowledge, it is the first time data from 10 of the trials included in this systematic review have been meta-analysed [32, 34, 102, 104, 108-110, 117, 119] suggesting that this is the most comprehensive and up-to-date systematic review on the topic of exercise in the treatment of women with PCOS. We followed the PRISMA statement [26] and used the PRISMA checklist (Additional file 3) to ensure methodological quality. Furthermore, we present our entire data set for transparency and reproducibility in Additional file 4.

However, there are limitations to this systematic review. It is likely that many of the included trials were not sufficiently powered to detect meaningful differences between test groups. Indeed, only seven included trials state the methods used to calculate sample size, and due to small participant numbers (e.g., median: exercise $n=$ 11 ; control $n=12$ ), it is unlikely that sufficient statistical power was achieved to either make the findings generalisable into the population or ensure that false positive/ negative results were not reported. Therefore, it is important that future trials are sufficiently powered to detect changes in their primary outcomes.
PCOS is a heterogeneous condition and can exhibit phenotypes with varying levels of underlying hyperandrogenism, menstrual disorders, and polycystic ovarian morphology [156]. It is likely that different phenotypes may respond differently to exercise and/or dietary interventions. Most included trials did not target a specific PCOS phenotype, and our protocol included a PCOS diagnosis based on any of the existing PCOS definitions/ criteria. Future work should focus on PCOS subgroups/ phenotypes and investigate the exercise-induced effects accordingly. Another concern surrounds the representativeness of the populations included in the review; it is not clear whether the ethnicity, socio-economic, or educational status of participants is representative of the typical patient or to what degree these variables may have influenced the observed effects.

All included trials reported baseline and immediately post-intervention data; only one trial [111] completed follow-up beyond the end of the intervention. Consequently, the lasting, long-term effect of exercise for women with PCOS is unknown. Future research is needed to determine whether behaviours relating to PA are changed in this patient population due to exercise interventions and whether the noted physiological effects remain beyond the short term.

\section{Quality of the evidence}

Due to the nature of the interventions, all included trials were judged to have a high risk of performance bias. All but one trial was judged to have a high detection bias risk due to lack of blinding outcome assessors, and although logistically difficult, steps could have been taken to minimise this bias in each trial. Selection and reporting bias were inadequately reported in $>50 \%$ of trials so a judgement of unclear risk was made and nearly $45 \%$ of the included trials $(n=8)$ were judged to be at a high risk of attrition bias. Six trials were at an unclear or high risk of baseline group imbalance, whereas adherence and contamination were generally unreported resulting in an unclear judgement. Disappointingly, few studies reported adherence data $(33 \%, n=6)$, but of the trials that did report these data, adherence rates were generally good (median 90\%). Similarly, in the 10 trials reporting attrition, the median value was $19.5 \%$; five of these were under the $20 \%$ attrition threshold outlined in the protocol.

Statistical effects were reported in 13 of the main analyses; in three of those, there was evidence of at least substantial heterogeneity $\left(I^{2} \geq 50 \%\right)$, but this was largely explained by subgroups and/or removal of trials with the most extreme values. For our primary outcomes, the quality of evidence was rated as very low to low due to a combination of unclear or high-risk randomisation or allocation procedures, lack of blinding, unclear or 
improper handling of missing data, high attrition, unclear risk of selective reporting bias, contamination, low adherence, or considerable heterogeneity. We downgraded all outcomes because of imprecision resulting from the small number of participants and either wide confidence intervals for the effect estimate or the null effect, as well as an appreciable benefit was included in the confidence interval for the mean difference.

\section{Limitations and potential biases in the review process}

In addition to the limitations mentioned in "Secondary outcomes" section, there are also further possible limitations to this systematic review. Despite a thorough and comprehensive search of relevant databases, we may have missed trials that would have been eligible for inclusion. Additionally, we did not identify any additional studies from the reference lists of the included publications; although this may support the comprehensiveness of our searches, it may also represent a methodological error. Also, no language restriction in our searches meant several foreign language papers were returned; three trials in Persian $[104,109,110]$ and one in Hungarian [107]. To assess these trials, translation services and software were required, and whilst interpretation of results tables was straightforward, evaluation of methodological quality was more challenging. Consequently, when assessing risk of bias in these trials, judgements of 'unclear risk' had to be made.

Finally, only full publications were eligible for inclusion and this could contribute to publication bias. Although including grey literature may have influenced the findings of this review, it may have also increased the risk of associated bias. Unfortunately, due to a lack of eligible trials, publication bias analysis was not performed.

\section{Future directions}

Based upon our findings, it is apparent that there is a lack of trials that compare exercise and diet combined with other comparators, such as diet only, exercise only, or a standard treatment control. Considering that lifestyle changes (i.e., diet and exercise) are recommended in the management of PCOS, studies assessing the effectiveness of these interventions are scarce and the available data are not sufficient to lead to definite conclusions/recommendations for the clinical practice. Future trials should aim to make comprehensive comparisons involving interventions that incorporate both exercise and diet.

Furthermore, the eligible studies included in the current systematic review generally have small sample sizes, whilst even those studies that have reported power calculations appear under-powered to detect meaningful changes in all reported outcomes. Therefore, it is important that future studies are robustly designed and sufficiently powered to better inform future clinical practice guidelines/recommendations. Considering the high prevalence of PCOS in reproductive-aged women, large RCTs studying the effectiveness of lifestyle interventions in this young patient population are still clearly needed.

We also identified a lack of follow-up testing beyond the intervention period to assess the longer-term effects of such lifestyle interventions. Without follow-up reassessments, it is impossible to determine whether any intervention-induced improvements are maintained, and if the applied intervention has resulted in sustained changes in lifestyle behaviours of participants, an aspect which is vital for the long-term management of these patients.

\section{Conclusion}

When data were pooled in a meta-analysis, changes from baseline statistically favoured exercise over control for FI, HOMA-IR, TC, LDL-C, triglycerides, $\mathrm{VO}_{2}$ max, WC, and body fat percentage. Furthermore, a comparison of immediately post-intervention values also revealed statistical effects on FI, TC, LDL-C, $\mathrm{VO}_{2}$ max, RHR, and BMI. Compared with control, exercise also improved the physical functioning, general health, social functioning, and mental health domains assessed in the SF-36. Subgroup analyses revealed that the greatest favourable changes with exercise versus control were seen in participants who were either overweight (FI, HOMA-IR, triglycerides, $\mathrm{VO}_{2}$ max, and WC) or obese (BMI, body mass, and WC). Post-intervention value analyses also showed beneficial effects in those who were overweight (LDL-C, $\mathrm{VO}_{2}$ max, RHR, BMI, WC, and body fat percentage). Aerobic exercise interventions improved FI, HOMA-IR, TC, triglycerides, $\mathrm{VO}_{2} \max , \mathrm{BMI}, \mathrm{WC}$, and body fat percentage. In contrast, resistance training lowered HDL-C concentrations and increased BMI, but reduced WC; post-intervention improvements in HDL-C were also apparent following resistance exercise. Supervised exercise interventions improved outcomes more than unsupervised interventions compared with control. Shorter duration interventions performed better than longer interventions; improved change from baseline FI, HOMA-IR, TC, LDL-C, triglycerides, $\mathrm{VO}_{2}$ max, and WC was found in shorter duration trials, compared with only improved $\mathrm{VO}_{2} \max$ in those $>12$ weeks. Based on limited available data, we found no differences between the effects of exercise and diet combined and diet alone. Due to lack of available trials, it was not possible to compare the effectiveness of exercise versus diet or exercise and diet combined versus diet.

Although the evidence presented within this systematic review has largely been drawn from RCTs, a cautious approach should be adopted when interpreting the findings. Many of the outcomes presented modest effects and wide confidence intervals (indicating greater uncertainty). Furthermore, we found the statistical effects in many of the 
analyses to be sensitive to the addition or removal of individual trials regardless of their weighting within the analysis. Using the GRADE approach, we rated the quality of evidence as very low or low for all primary outcomes. Future trials should be rigorously designed and sufficiently powered so that they are more generalizable to the wider PCOS population. In order to be more closely aligned with current treatment recommendations, future studies should ideally include a dietary component alongside exercise interventions.

\section{Additional files}

\begin{abstract}
Additional file 1: Table S1. Search algorithm; Table S2. Review authors' judgement on risk of bias; Table S3. Details of excluded studies and reasons for exclusion; Table S4. Diagnostic criteria/definition applied for PCOS; Table S5 Effect estimates and heterogeneity from subgroup analyses in blood pressure and metabolism-related outcomes; Table S6. Effect estimates and heterogeneity from subgroup analyses in lipid-related outcomes; Table S7. Effect estimates and heterogeneity from subgroup analyses in cardiorespiratory, anthropometric and body composition related outcomes; Table S8. Effect estimates and heterogeneity from subgroup analyses in androgenic and inflammatory outcomes; Table S9. Exercise vs control-Summary of findings from investigative outcomes that were only reported in single trials; Table S10. Exercise and diet vs control-Summary of findings from investigative outcomes that were only reported in single trials; Table S11. Effect estimates and heterogeneity for change from baseline to immediately post-intervention, and immediately post-intervention values only, for all outcomes analysed in the comparison exercise and diet versus diet only; Table S12. Exercise and diet vs diet-summary of findings from investigative outcomes that were only reported in single trials (PDF $296 \mathrm{~kb}$ )
\end{abstract}

Additional file 2: Figure S1. Review of authors' judgements about each risk of bias item for each included study; Figure S2. Forest plot of comparison: exercise vs. control. Analysis of immediately postintervention values for outcomes related to participant lipid profile; Figure S3. Forest plot of comparison: exercise vs. control. Analysis of resting heart rate subgroups by intervention type; Figure S4. Forest plot of comparison: exercise vs. control, change from baseline; outcome: SF-36 domains; Figure S5. Forest plot of comparison: exercise and diet vs. control; outcome: waist-to-hip ratio; Figure S6. Forest plot of comparison: exercise and diet vs. control, change from baseline. outcome: SHBG. (PDF $345 \mathrm{~kb}$ )

Additional file 3: PRISMA Checklist (PDF $202 \mathrm{~kb}$ )

Additional file 4: Additional meta-analyses/complete dataset. Supplementary meta-analyses: exercise vs. control; exercise and diet versus diet. (PDF 913 kb)

\footnotetext{
Abbreviations

AMH: Anti-Mullerian hormone; BMI: Body mass index; CHD: Coronary heart disease; Cls: Confidence intervals; CRF: Cardiorespiratory fitness;

CVD: Cardiovascular disease; DBP: Diastolic blood pressure; DHEAS: Dehydroepiandrosterone sulfate; FAl: Free androgen index; FBG: Fasting blood glucose; FFM: Fat-free mass; FG: Ferriman-Gallwey score; Fl: Fasting insulin; FSH: Follicle stimulating hormone; HDL-C: High-density lipoprotein cholesterol; HOMA-IR: Homeostatic model assessment of insulin resistance index; HR: Heart rate; $\mathrm{HR}_{\text {max }}$ : Maximum heart rate; HRQoL: Health-related quality of life; hsCRP: High-sensitivity C-reactive protein; IR: Insulin resistance; LDL-C: Low-density lipoprotein cholesterol; LH: Luteinising hormone; MD: Mean difference; MET: Metabolic equivalent of task; PA: Physical activity; PCOS: Polycystic ovary syndrome; PCOS-Q: Polycystic ovary syndrome questionnaire; RCT: Randomised controlled clinical trials; RHR: Resting heart rate; SBP: Systolic blood pressure; SD: Standard deviation; SF-36: 36-item Short Form Survey; SHBG: Sex hormone binding globulin; SMD: Standardised mean difference; T2DM: Type 2 diabetes mellitus; TC: Total cholesterol; $\mathrm{VO}_{2}$ max: Maximal oxygen uptake; WC: Waist circumference; WHR: Waist-to-hip ratio
}

\section{Acknowledgements}

Not Applicable.

\section{Funding}

This systematic review was completed as part of a funded PhD project (CK) through Aston University. No external funding was received.

\section{Availability of data and materials}

The datasets generated and analysed during this study are included in this published article (or within the supplementary information files.

\section{Authors' contributions}

CK designed the study, completed the database searches, extracted and analysed the data, and developed, revised, and edited the final manuscript. IML designed the study, analysed the data, and revised and edited the fina manuscript. IA, DRB, and IK analysed the data and revised and edited the final manuscript. HR revised and edited the final manuscript. JEB designed the study and revised and edited the final manuscript. All authors read and approved the final manuscript.

\section{Authors' information}

CK is a PhD research student; IML is a senior lecturer in sport and exercise physiology; IA is a medical researcher; DRB is a senior lecturer in physical activity, health and exercise science; HR is a professor in the study of diabetes, endocrinology and metabolism; IK is a clinical lecturer in metabolic medicine and nutrition; JEB is a senior lecturer in biology and biomedical science.

Ethics approval and consent to participate

Not Applicable.

\section{Consent for publication}

Not Applicable.

\section{Competing interests}

The authors declare that they have no competing interests.

\section{Publisher's Note}

Springer Nature remains neutral with regard to jurisdictional claims in published maps and institutional affiliations.

\section{Author details}

${ }^{1}$ Aston Medical Research Institute, Aston Medical School, Aston University, Birmingham B4 7ET, UK. ${ }^{2}$ Division of Translational and Experimental Medicine, Warwick Medical School, University of Warwick, Coventry CV4 7AL, UK. ${ }^{3}$ Warwickshire Institute for the Study of Diabetes, Endocrinology and Metabolism (WISDEM), University Hospitals Coventry and Warwickshire NHS Trust, Coventry CV2 2DX, UK. ${ }^{4}$ Centre of Applied Biological \& Exercise Sciences, Faculty of Health \& Life Sciences, Coventry University, Coventry CV1 5FB, UK. ${ }^{5}$ School of Life and Health Sciences, Cell and Tissue Biomedical Research Group, Aston University, Aston Triangle, Birmingham B4 7ET, UK. ${ }^{6}$ Faculty of Education Health and Wellbeing, University of Wolverhampton, Walsall Campus, Gorway Road, Walsall WS1 3BD, UK. ${ }^{7}$ Centre for Active Living, University Centre Shrewsbury, University of Chester, Guildhall, Frankwell Quay, Shrewsbury SY3 8HQ, UK. ${ }^{8}$ Academy of Sport and Physical Activity, Faculty of Health and Wellbeing, Sheffield Hallam University, Sheffield S10 2BP, UK.

Received: 30 August 2018 Accepted: 23 January 2019

Published online: 12 February 2019

\section{References}

1. Boyle JA, Cunningham J, O'Dea K, Dunbar T, Norman RJ. Prevalence of polycystic ovary syndrome in a sample of indigenous women in Darwin. Australia Med J Aust. 2012;196(1):62-6.

2. Lizneva D, Suturina L, Walker W, Brakta S, Gavrilova-Jordan L, Azziz R. Criteria, prevalence, and phenotypes of polycystic ovary syndrome. Fertil Steril. 2016;106(1):6-15.

3. Ma YM, Li R, Qiao J, Zhang XW, Wang SY, Zhang QF, Li L, Tu BB, Zhang X. Characteristics of abnormal menstrual cycle and polycystic ovary syndrome 
in community and hospital populations. Chinese Med J-Peking. 2010; 123(16):2185-9.

4. Costello MF, Shrestha B, Eden J, Johnson N, Moran LJ. Insulin-sensitising drugs versus the combined oral contraceptive pill for hirsutism, acne and risk of diabetes, cardiovascular disease, and endometrial cancer in polycystic ovary syndrome. Cochrane Database Syst Rev. 2007;1(CD005552).

5. Azziz R, Carmina E, Chen Z, Dunaif A, Laven JS, Legro RS, Yildiz BO. Polycystic ovary syndrome. Nat Rev Dis Primers. 2016;2:16057.

6. Himelein MJ, Thatcher SS. Depression and body image among women with polycystic ovary syndrome. J Health Psychol. 2006;11(4):613-25.

7. Weiner CL, Primeau M, Ehrmann DA. Androgens and mood dysfunction in women: comparison of women with polycystic ovarian syndrome to healthy controls. Psychosom Med. 2004;66(3):356-62.

8. Sam S. Obesity and polycystic ovary syndrome. Obes Manag. 2007;3(2):69-73.

9. Sir-Petermann T, Codner E, Pérez V, Echiburú B, Maliqueo M, Ladrón de Guevara A, Preisler J, Crisosto N, Sánchez F, Cassorla F, Bhasin S. Metabolic and reproductive features before and during puberty in daughters of women with polycystic ovary syndrome. J Clin Endocrinol Metab. 2009;94:1923-30.

10. Balen AH, Conway GS, Kaltsas G, Techatraisak K, Manning PJ, West C, Jacobs HS. Andrology: polycystic ovary syndrome: the spectrum of the disorder in 1741 patients. Hum Reprod. 1995;10(8):2107-11.

11. Legro RS. The genetics of obesity: lessons for polycystic ovary syndrome. Ann N Y Acad Sci. 2000;900:193-202.

12. Kiddy DS, Hamilton-Fairley D, Bush A, Short F, Anyaoku V, Reed MJ, Franks S. Improvement in endocrine and ovarian function during dietary treatment of obese women with polycystic ovary syndrome. Clin Endocrinol. 1992;36: 105-11.

13. Holte J, Bergh T, Berne C, Wide L, Lithell H. Restored insulin sensitivity but persistently increased early insulin secretion after weight loss in obese women with polycystic ovary syndrome. J Clin Endocr Metab. 1995;80(9): 2586-93.

14. Dunaif A, Segal KR, Futterweit W, Dobrjansky A. Profound peripheral insulin resistance, independent of obesity, in polycystic ovary syndrome. Diabetes. 1989:38(9):1165-74.

15. Norman RJ, Masters L, Milner CR, Wang JX, Davies MJ. Relative risk of conversion from normoglycaemia to impaired glucose tolerance or noninsulin dependent diabetes mellitus in polycystic ovarian syndrome. Hum Reprod. 2001:16(9):1995-8.

16. Glueck CJ, Dharashivkar S, Wang P, Zhu B, Gartside PS, Tracy T, Sieve L. Obesity and extreme obesity, manifest by ages 20-24 years, continuing through 32-41 years in women, should alert physicians to the diagnostic likelihood of polycystic ovary syndrome as a reversible underlying endocrinopathy. Eur J Obstet Gyn R B. 2005;122(2):206-12.

17. Legro RS, Kunselman AR, Dunaif A. Prevalence and predictors of dyslipidemia in women with polycystic ovary syndrome. Am J Med. 2001; 111(8):607-13.

18. Ehrmann DA, Liljenquist DR, Kasza K, Azziz R, Legro RS, Ghazzi MN Prevalence and predictors of the metabolic syndrome in women with polycystic ovary syndrome. J Clin Endocr Metab. 2006;91(1):48-53.

19. Kiddy DS, Sharp PS, White DM, Scanlon MF, Mason HD, Bray CS, Franks S. Differences in clinical and endocrine features between obese and nonobese subjects with polycystic ovary syndrome: an analysis of 263 consecutive cases. Clin Endocrinol. 1990;32(2):213-20.

20. Legro RS, Kunselman AR, Dodson WC, Dunaif A. Prevalence and predictors of risk for type 2 diabetes mellitus and impaired glucose tolerance in polycystic ovary syndrome: a prospective, controlled study in 254 affected women. J Clin Endocr Metab. 1999;84(1):165-9.

21. National Institute for Health and Care Excellence. Scenario: management of polycystic ovary syndrome. 2013. https://cks.nice.org.uk/polycystic-ovarysyndrome\#!scenariorecommendation:5. Accessed 30 Jan 2019.

22. Boulé NG, Haddad E, Kenny GP, Wells GA, Sigal RJ. Effects of exercise on glycemic control and body mass in type 2 diabetes mellitus: a meta-analysis of controlled clinical trials. JAMA. 2001;286(10):1218-27.

23. Thomas DE, Elliott EJ, Naughton GA. Exercise for type 2 diabetes mellitus. Cochrane Database Syst Rev. 2006;3(CD002968).

24. Yang Z, Scott CA, Mao C, Tang J, Farmer AJ. Resistance exercise versus aerobic exercise for type 2 diabetes: a systematic review and meta-analysis. Sports Med. 2014;44(4):487-99.

25. Harrison $\mathrm{CL}$, Lombard CB, Moran LJ, Teede HJ. Exercise therapy in polycystic ovary syndrome: a systematic review. Hum Reprod Update. 2011;17(2):171-83.
26. Moher D, Liberati A, Tetzlaff J, Altman DG. The PRISMA group (2009) Preferred Reporting Items for Systematic Reviews and Meta-Analyses: the PRISMA statement. PLoS Med. 2009;6(7):e1000097.

27. Winter E, Fowler N. Exercise defined and quantified according to the Système International d'Unités. J Sport Sci. 2009;27(5):447-60.

28. Higgins JPT, Altman DG. Assessing risk of bias in included studies. In: Higgins JPT, Green S, editors. Cochrane Handbook for Systematic Reviews of Interventions. Chichester: The Cochrane Collaboration and John Wiley and Sons Ltd; 2011. p. 187-241.

29. Furmaniak AC, Menig M, Markes MH. Exercise for women receiving adjuvant therapy for breast cancer. Cochrane Database Syst Rev. 2016;9:CD005001.

30. Deeks JJ, Higgins JPT, Altman DG. Analysing data and undertaking metaanalyses. In: Higgins JPT, Green S, editors. Cochrane Handbook for Systematic Reviews of Interventions. Chichester: The Cochrane Collaboration and John Wiley and Sons Ltd; 2011. p. 243-96.

31. DerSimonian R, Laird N. Meta-analysis in clinical trials. Control Clin Trials. 1986;7:177-88.

32. Almenning I, Rieber-Mohn A, Lundgren KM, Løvvik TS, Garnæs KK, Moholdt $T$. Effects of high intensity interval training and strength training on metabolic, cardiovascular and hormonal outcomes in women with polycystic ovary syndrome: a pilot study. PLoS One. 2015;10(9):e0138793.

33. Thomson RL, Buckley JD, Noakes M, Clifton PM, Norman RJ, Brinkworth GD. The effect of a hypocaloric diet with and without exercise training on body composition, cardiometabolic risk profile, and reproductive function in overweight and obese women with polycystic ovary syndrome. J Clin Endocr Metab. 2008:93(9):3373-80.

34. Roessler KK, Birkebaek C, Ravn P, Andersen MS, Glintborg D. Effects of exercise and group counselling on body composition and $\mathrm{VO}_{2} \max$ in overweight women with polycystic ovary syndrome. Acta Obstet Gyn Scan. 2013;92(3):272-7.

35. Schünemann H, Brożek J, Guyatt G, Oxman A, editors. GRADE handbook for grading quality of evidence and strength of recommendations. Updated October 2013. The GRADE Working Group.

36. Costa EC, Sá JC, Stepto NK, Costa IBB, Farias-Junior LF, Moreira SNT, Soares EMM, Lemos TMAM, Browne RAV, Azevedo GD. Aerobic training improves quality of life in women with polycystic ovary syndrome. Med Sci Sport Exer. 2018;50(7):1357-66

37. Almenning I, Rieber-mohn A, Garnaes KK, Lundgren KM, Lovik TS, Moholdt $\Pi$. High intensity interval training versus strength training to improve cardiovascular risk factors in women with polycystic ovary syndrome. A randomized controlled trial. Eur J Prev Cardiol. 2015;22:S23.

38. Asante A, Konopka AR, Stewart EA, Coddington CC, Nair SK. Impact of aerobic exercise on mitochondrial function and insulin sensitivity in women with polycystic ovary syndrome-a randomized controlled trial. Reprod Sci. 2014;21(3):126A

39. Bachani S, Malla VG, Singh P. M110 to study the efficacy of intervention strategies on features of metabolic syndrome in Asian urban adolescent girls with PCOS. Int J Gynecol Obstet. 2012;119:S567.

40. Barr S, Hart K, Reeves S, Sharp K, Jeanes YM. Habitual dietary intake, eating pattern and physical activity of women with polycystic ovary syndrome. Eur J Clin Nutr. 2011;65(10):1126-32.

41. Bongaard BS. Fact or fiction? Is a low glycemic index diet the best choice for your PCOS patients? Alternative Med Alert. 2010;13(8):91-3.

42. Brown AJ, Aiken LB, Setji T, Sanders L, Kraus WE, Svetkey LP. Effect of exercise without weight loss on insulin resistance in women with polycystic ovary syndrome: a randomized controlled study, 3rd Annual Meeting of the Androgen Excess Society, vol. 28; 2005.

43. Christiansen SC, Vanky E, Klungland H, Stafne SN, Mørkved S, Salvesen KÅ, Carlsen SM. The effect of exercise and metformin treatment on circulating free DNA in pregnancy. Placenta. 2014;35(12):989-93.

44. Chizen DR, Serrao S, Rooke J, McBreairty L, Pierson RA, Chilibeck P, Zello G. The "pulse" diet \& PCOS. Fertil Steril. 2014;102(3):e267.

45. Crosignani PG, Colombo M, Vegetti W, Somigliana E, Gessati A, Ragni G. Overweight and obese anovulatory patients with polycystic ovaries: parallel improvements in anthropometric indices, ovarian physiology and fertility rate induced by diet. Hum Reprod. 2003;18(9):1928-32.

46. Curi DD, Fonseca AM, Marcondes JAM, Almeida JAM, Bagnoli VR, Soares JM $\mathrm{Jr}$, Baracat EC. Metformin versus lifestyle changes in treating women with polycystic ovary syndrome. Gynecol Endocrinol. 2012;28(3):182-5.

47. Fux Otta C, Wior M, Iraci GS, Kaplan R, Torres D, Gaido MI, Wyse EP. Clinical, metabolic, and endocrine parameters in response to metformin 
and lifestyle intervention in women with polycystic ovary syndrome: a randomized, double-blind, and placebo control trial. Gynecol Endocrinol. 2010;26(3):173-8.

48. Galletly C, Moran L, Noakes M, Clifton P, Tomlinson L, Norman R. Psychological benefits of a high-protein, low-carbohydrate diet in obese women with polycystic ovary syndrome-a pilot study. Appetite. 2007; 49(3):590-3.

49. Gambineri A, Patton L, Vaccina A, Cacciari M, Morselli-Labate AM, Cavazza C, Pasquali R. Treatment with flutamide, metformin, and their combination added to a hypocaloric diet in overweight-obese women with polycystic ovary syndrome: a randomized, 12-month, placebo-controlled study. J Clin Endocr Metab. 2006;91(10):3970-80.

50. Giallauria F, Palomba S, Maresca L, Vuolo L, Tafuri D, Lombardi G, Vigorito $C$. Exercise training improves autonomic function and inflammatory pattern in women with polycystic ovary syndrome (PCOS). Clin Endocrinol. 2008;69(5):792-8.

51. Harris-Glocker M, Davidson K, Kochman L, Guzick D, Hoeger K. Improvement in quality-of-life questionnaire measures in obese adolescent females with polycystic ovary syndrome treated with lifestyle changes and oral contraceptives, with or without metformin. Fertil Steril. 2010;93(3):1016-9.

52. Hoeger K, Davidson K, Kochman L, Cherry T, Kopin L, Guzick DS. The impact of metformin, oral contraceptives, and lifestyle modification on polycystic ovary syndrome in obese adolescent women in two randomized, placebocontrolled clinical trials. J Clin Endocr Metab. 2008:93(11):4299-306.

53. Jaffe RB. Combined lifestyle modification and metformin in obese patients with polycystic ovary syndrome: a randomized, placebo-controlled, doubleblind multicentre study. Obstet Gynecol Surv. 2006;61(2):108-9.

54. Jedel E, Holm G, Labrie F, Oden A, Nilsson L, Janson PO, Stener-Victorin E. Effect of low-frequency electro-acupuncture on serum testosterone and menstrual pattern in women with polycystic ovary syndrome compared to physical exercise: randomised controlled trial. In: Endocrine Society Posters IX, vol. P2; 2010. p. 410

55. Johansson J, Redman L, Veldhuis PP, Sazonova A, Labrie F, Holm G, StenerVictorin E. Acupuncture for ovulation induction in polycystic ovary syndrome: a randomized controlled trial. Am J Physiol-Endoc M. 2013;304(9):E934-43.

56. Karimzadeh MA, Javedani M. An assessment of lifestyle modification versus medical treatment with clomiphene citrate, metformin, and clomiphene citrate-metformin in patients with polycystic ovary syndrome. Fertil Steril. 2010;94(1):216-20.

57. Kumar $\mathrm{P}$, Arora $\mathrm{S}$. Orlistat in polycystic ovarian syndrome reduces weight with improvement in lipid profile and pregnancy rates. J Hum Reprod Sci. 2014;7(4):255.

58. Ladson G, Dodson WC, Sweet SD, Archibong AE, Kunselman AR, Demers LM, Legro RS. Effects of metformin in adolescents with polycystic ovary syndrome undertaking lifestyle therapy: a pilot randomized double-blind study. Fertil Steril. 2011;95(8):2595-8

59. Ladson G, Dodson WC, Sweet SD, Archibong AE, Kunselman AR, Demers LM, Legro RS. The effects of metformin with lifestyle therapy in polycystic ovary syndrome: a randomized double-blind study. Fertil Steril. 2011;95(3): 1059-66.

60. Le Donne M, Alibrandi A, Giarrusso R, Lo MI, Muraca U. Diet, metformin and inositol in overweight and obese women with polycystic ovary syndrome: effects on body composition. Minerva Ginecol. 2012;64(1):23-9.

61. Legro RS, Dodson WC, Kunselman AR, Kris-Etherton PM, Allison KC, Sarwer $D B$, Coutifaris C. Effects of preconception intervention on the PCOS phenotype, ovulation, and live birth rates: a multi-center, multi-phase RCT. Fertil Steril. 2014;102(3):e2.

62. Legro RS, Dodson WC, Kris-Etherton PM, Kunselman AR, Stetter CM, Williams $\mathrm{NI}$, Sarwer DB. Randomized controlled trial of preconception interventions in infertile women with polycystic ovary syndrome. J Clin Endocr Metab. 2015;100(11):4048-58.

63. Liao LM, Nesic J, Chadwick PM, Brooke-Wavell K, Prelevic GM. Exercise and body image distress in overweight and obese women with polycystic ovary syndrome: a pilot investigation. Gynecol Endocrinol. 2008;24(10):555-61.

64. Lindholm Å, Bixo M, Björn I, Wölner-Hanssen P, Eliasson M, Larsson A, Poromaa IS. Effect of sibutramine on weight reduction in women with polycystic ovary syndrome: a randomized, double-blind, placebo-controlled trial. Fertil Steril. 2008;89(5):1221-8.

65. Ma LK, Jin LN, Yu Q, Xu L. Effect of lifestyle adjustment, metformin and rosiglitazone in polycystic ovary syndrome. Zhonghua fu chan ke za zhi. 2007:42(5):294-7.
66. Machlitt AR, Steinheim E, Pfueller BF. Effects of metformin plus lifestyle modification on menstrual cycle and body mass index are not influenced by insulin resistance: a 16 week, double-blind, controlled, randomised study in PCOS. Fertil Steril. 2007:88:S15-6.

67. Marzouk TM, Ahmed WAS. Effect of dietary weight loss on menstrual regularity in obese young adult women with polycystic ovary syndrome. J Pediatr Adolesc Gynecol. 2015;28(6):457-61.

68. McBreairty L, Zello G, Rooke J, Serrao S, Pierson R, Chizen D, Chilibeck P. Long-term effect of a pulse-based diet and exercise training intervention on body composition and dietary intake in women with polycystic ovarian syndrome. FASEB J. 2015;29(1):912-5.

69. Mehrabani HH, Salehpour S, Amiri Z, Farahani SJ, Meyer BJ, Tahbaz F. Beneficial effects of a high-protein, low-glycemic-load hypocaloric diet in overweight and obese women with polycystic ovary syndrome: a randomized controlled intervention study. J Am Coll Nutr. 2012;31(2):117-25.

70. Moran LJ, Noakes M, Norman RJ, Clifton PM, Tomlinson L. Dietary composition and weight loss in improving reproductive and metabolic physiology in overweight women with polycystic ovary syndrome. Asia Pac J Clin Nutr. 2002;11:S271.

71. Moran LJ, Noakes M, Clifton PM, Tomlinson L, Norman RJ. Dietary composition in restoring reproductive and metabolic physiology in overweight women with polycystic ovary syndrome. J Clin Endocr Metab. 2003:88(2):812-9.

72. Moran L, Noakes M, Clifton PM, Wittert GA, Williams G, Norman RJ. Short-term meal replacements followed by dietary macronutrient restriction enhance weight loss in polycystic ovary syndrome. Am J Clin Nutr. 2006;84(1):77-87.

73. Nidhi R, Padmalatha $V$, Nagarathna R, Ram A. Effect of a yoga program on glucose metabolism and blood lipid levels in adolescent girls with polycystic ovary syndrome. Int J Gynecol Obstet. 2012;118(1):37-41.

74. Nidhi R, Padmalatha V, Nagarathna R, Amritanshu R. Effects of a holistic yoga program on endocrine parameters in adolescents with polycystic ovarian syndrome: a randomized controlled trial. J Altern Complem Med. 2013;19(2):153-60

75. Nidhi $R$, Padmalatha $V$, Nagarathna $R$, Amritanshu R. Effect of yoga program on quality of life in adolescent polycystic ovarian syndrome: a randomized control trial. Appl Res Qual Life. 2013:8(3):373-83.

76. Nybacka A, Hellstrom PM, Hirschberg AL. Nutritional counseling significantly improves metabolic variables in overweight/obese women with polycystic ovary syndrome. Clin Nutr. 2014;33:S246-S247.

77. Nybacka A, Fabri F, Hellstrom PM, Hirschberg AL, Stahle A. Plasma antiMullerian hormone $(\mathrm{AMH})$ levels correlate and predict the response to lifestyle intervention in polycystic ovarian syndrome. Clin Nutr. 2012;S7(1):255.

78. Omar AN, Habib F, Mojaddidi M, El-Bab MF. Body weight reduction and metformin: roles in polycystic ovary syndrome. Pathophysiology. 2013; 20(2):131-7

79. Orio F, Muscogiuri G, Giallauria F, Savastano S, Bottiglieri P, Tafuri D, Palomba S. Oral contraceptives versus physical exercise on cardiovascular and metabolic risk factors in women with polycystic ovary syndrome: a randomized controlled trial. Clin Endocrinol. 2016;85(5):764-71.

80. Ornstein RM, Copperman NM, Jacobson MS. Effect of weight loss on menstrual function in adolescents with polycystic ovary syndrome. J Pediatr Adolesc Gynecol. 2011;24(3):161-5.

81. Palomba S, Giallauria F, Falbo A, Russo T, Oppedisano R, Tolino A, Orio F. Structured exercise training programme versus hypocaloric hyperproteic diet in obese polycystic ovary syndrome patients with anovulatory infertility: a 24-week pilot study. Hum Reprod. 2007;23(3):642-50.

82. Palomba S, Falbo A, Giallauria F, Russo T, Rocca M, Tolino A, Orio F. Six weeks of structured exercise training and hypocaloric diet increases the probability of ovulation after clomiphene citrate in overweight and obese patients with polycystic ovary syndrome: a randomized controlled trial. Hum Reprod. 2010;25(11):2783-91.

83. Papakonstantinou E, Kechribari I, Mitrou P, Trakakis E, Vassiliadi D, Georgousopoulou E, Dimitriadis G. Effect of meal frequency on glucose and insulin levels in women with polycystic ovary syndrome: a randomised trial. Eur J Clin Nutr. 2016;70(5):588-94.

84. Pasquali R, Fabbri R, Venturoli S, Paradisi R, Antenucci D, Melchionda N. Effect of weight loss and antiandrogenic therapy on sex hormone blood levels and insulin resistance in obese patients with polycystic ovaries. Am J Obstet Gynecol. 1986;154(1):139-44.

85. Pasquali R, Gambineri A, Biscotti D, Vicennati V, Gagliardi L, Colitta D, Morselli-Labate AM. Effect of long-term treatment with metformin added to 
hypocaloric diet on body composition, fat distribution, and androgen and insulin levels in abdominally obese women with and without the polycystic ovary syndrome. J Clin Endocr Metab. 2000;85(8):2767-74.

86. Popova P, Ryasantseva E, Zazerskaya I, Ivanova L, Grineva E. The role of weight loss and metformin in the improvement of menstrual function in overweight and obese women with polycystic ovary syndrome. Diabetes Obes Metab. 2010;12:87.

87. Randeva HS, Lewandowski KC, Drzewoski J, Brooke-Wavell K, O'Callaghan C, Czupryniak L, Prelevic GM. Exercise decreases plasma total homocysteine in overweight young women with polycystic ovary syndrome. J Clin Endocr Metab. 2002;87(10):4496-501.

88. Redman LM, Elkind-Hirsch K, Ravussin E. Aerobic exercise in women with polycystic ovary syndrome improves ovarian morphology independent of changes in body composition. Fertil Steril. 2011;95(8):2696-9.

89. Roessler KK, Andersen M, Glintborg D, Ravn P, Birkebaek C. F417 group counselling and physical activity of patients with polycystic ovary syndrome (PCOS). Eur J Pain Suppl. 2011;5(1):156.

90. Silva Dantas W, Antonio Miguel Marcondes J, Katsuyuki Shinjo S, Augusto Perandini L, Olzon Zambelli V, Das Neves W, Hisashi Murai I. GLUT4 translocation is not impaired after acute exercise in skeletal muscle of women with obesity and polycystic ovary syndrome. Obesity. 2015;23(11): 2207-15.

91. Sørensen LB, Søe M, Halkier KH, Stigsby B, Astrup A. Effects of increased dietary protein-to-carbohydrate ratios in women with polycystic ovary syndrome. Am J Clin Nutr. 2011;95(1):39-48.

92. Tang T, Glanville J, Hayden CJ, White D, Barth JH, Balen AH. Combined lifestyle modification and metformin in obese patients with polycystic ovary syndrome. A randomized, placebo-controlled, double-blind multicentre study. Hum Reprod. 2005;21(1):80-9.

93. Thomson RL, Buckley JD, Moran L, Noakes M, Clifton PM, Norman RJ, Brinkworth GD. The effect of weight loss on anti-Müllerian hormone levels in overweight and obese women with polycystic ovary syndrome and reproductive impairment. Hum Reprod. 2009;24(8):1976-81.

94. Thomson RL, Brinkworth GD, Noakes M, Clifton PM, Norman RJ, Buckley JD. The effect of diet and exercise on vascular function in overweight and obese women with polycystic ovary syndrome. Obes Res Clin Pract. 2011;5:15.

95. Turner-McGrievy GM, Davidson CR, Wingard EE, Billings DL. Low glycemic index vegan or low-calorie weight loss diets for women with polycystic ovary syndrome: a randomized controlled feasibility study. Nutr Res. 2014; 34(6):552-8.

96. Turner-McGrievy G, Davidson CR, Billings DL. Dietary intake, eating behaviors, and quality of life in women with polycystic ovary syndrome who are trying to conceive. Hum Fertil. 2015;18(1):16-21.

97. Brown AJ, Setji TL, Sanders LL, Lowry KP, Otvos JD, Kraus WE, Svetkey LP. Effects of exercise on lipoprotein particles in women with polycystic ovary syndrome. Med Sci Sport Exer. 2009;41(3):497.

98. Bruner B, Chad K, Chizen D. Effects of exercise and nutritional counselling in women with polycystic ovary syndrome. Appl Physiol Nutr Metab. 2006; 31(4):384-91.

99. Guzick DS, Wing R, Smith D, Berga SL, Winters SJ. Endocrine consequences of weight loss in obese, hyperandrogenic, anovulatory women. Fertil Steril. 1994;61(4):598-604.

100. Hoeger KM, Kochman L, Wixom N, Craig K, Miller RK, Guzick DS. A randomized, 48-week, placebo-controlled trial of intensive lifestyle modification and/or metformin therapy in overweight women with polycystic ovary syndrome: a pilot study. Fertil Steril. 2004;82(2):421-9.

101. Jedel E, Labrie F, Odén A, Holm G, Nilsson L, Janson PO, Stener-Victorin E. Impact of electro-acupuncture and physical exercise on hyperandrogenism and oligo/amenorrhea in women with polycystic ovary syndrome: a randomized controlled trial. Am J Physiol-Endoc M. 2011;300(1):E37-45.

102. Konopka AR, Asante A, Lanza IR, Robinson MM, Johnson ML, Dalla Man C, Nair KS. Defects in mitochondrial efficiency and $\mathrm{H}_{2} \mathrm{O}_{2}$ emissions in obese women are restored to a lean phenotype with aerobic exercise training. Diabetes. 2015;64(6):2104-15.

103. Leonhardt H, Hellström M, Gull B, Lind AK, Nilsson L, Janson PO, StenerVictorin E. Serum anti-Müllerian hormone and ovarian morphology assessed by magnetic resonance imaging in response to acupuncture and exercise in women with polycystic ovary syndrome: secondary analyses of a randomized controlled trial. Acta Obstet Gyn Scan. 2015;94(3):279-87.

104. Nasrekani Z, Fathi M. Efficacy of 12 weeks aerobic training on body composition, aerobic power and some women-hormones in polycystic ovary syndrome infertile women. [Persian]. Iran J Obstet Gynecol Infertil. 2016;19:1-10.

105. Nybacka Å, Carlström K, Ståhle A, Nyrén S, Hellström PM, Hirschberg AL. Randomized comparison of the influence of dietary management and/or physical exercise on ovarian function and metabolic parameters inoverweight women with polycystic ovary syndrome. Fertil Steril. 2011; 96(6): 1508-13.

106. Nybacka $\AA$, Carlström K, Fabri F, Hellström PM, Hirschberg AL. Serum antimüllerian hormone in response to dietary management and/or physical exercise in overweight/obese women with polycystic ovary syndrome: secondary analysis of a randomized controlled trial. Fertil Steril. 2013;100(4): 1096-102.

107. Petrányi G, Zaoura-Petrányi M. Metformin treatment with or without life style changes in patients with polycystic ovary syndrome. Orv Hetil. 2011; 152(16):628-32.

108. Sá JC, Costa EC, Da Silva E, Tamburús NY, Porta A, Medeiros LF, Azevedo GD. Aerobic exercise improves cardiac autonomic modulation in women with polycystic ovary syndrome. Int J Cardiol. 2015;202:356-61.

109. Saremi A, Shavandi N, Karamali M, Kazemi M. Serum level of anti-mullerian hormone after exercise training in women with polycystic ovary syndrome: a randomized controlled trial. Iran J Obstet Gynecol Infertil. 2013;16(64):10-8.

110. Saremi A, Yaghoubi MS. Effect of resistance exercises with calcium consumption on level of anti-mullerian hormone and some metabolic indices in women with polycystic ovarian syndrome. Iran J Obstet Gynecol Infertil. 2016;18(180):7-15.

111. Stener-Victorin $E$, Jedel $E$, Janson PO, Sverrisdottir YB. Low-frequency electroacupuncture and physical exercise decrease high muscle sympathetic nerve activity in polycystic ovary syndrome. Am J Physiol-Reg I. 2009;297(2):R387-95.

112. Stener-Victorin E, Baghaei F, Holm G, Janson PO, Olivecrona G, Lönn M, Mannerås-Holm L. Effects of acupuncture and exercise on insulin sensitivity, adipose tissue characteristics, and markers of coagulation and fibrinolysis in women with polycystic ovary syndrome: secondary analyses of a randomized controlled trial. Fertil Steril. 2012;97(2):501-8.

113. Stener-Victorin E, Holm G, Janson PO, Gustafson D, Waern M. Acupuncture and physical exercise for affective symptoms and health-related quality of life in polycystic ovary syndrome: secondary analysis from a randomized controlled trial. BMC Complement Altern Med. 2013;13(1):131.

114. Thomson RL, Buckley JD, Lim SS, Noakes M, Clifton PM, Norman RJ, Brinkworth GD. Lifestyle management improves quality of life and depression in overweight and obese women with polycystic ovary syndrome. Fertil Steril. 2010;94(5):1812-6.

115. Thomson RL, Brinkworth GD, Noakes M, Clifton PM, Norman RJ, Buckley JD. The effect of diet and exercise on markers of endothelial function in overweight and obese women with polycystic ovary syndrome. Hum Reprod. 2012;27(7):2169-76.

116. Thomson RL, Buckley JD, Brinkworth GD. Perceived exercise barriers are reduced and benefits are improved with lifestyle modification in overweight and obese women with polycystic ovary syndrome: a randomised controlled trial. BMC Womens Health. 2016;16(1):14.

117. Turan V, Mutlu EK, Solmaz U, Ekin A, Tosun O, Tosun G, Malkoc M. Benefits of short-term structured exercise in non-overweight women with polycystic ovary syndrome: a prospective randomized controlled study. J Phys Ther Sci. 2015;27(7):2293-7.

118. Vigorito C, Giallauria F, Palomba S, Cascella T, Manguso F, Lucci R, Orio F. Beneficial effects of a three-month structured exercise training program on cardiopulmonary functional capacity in young women with polycystic ovary syndrome. J Clin Endocrinol Metab. 2007;92(4):1379-84.

119. Vizza L, Smith CA, Swaraj S, Agho K, Cheema BS. The feasibility of progressive resistance training in women with polycystic ovary syndrome: a pilot randomized controlled trial. BMC Sports Sci Med Rehabil. 2016;8(1):14.

120. Zawadski JK, Dunaif A. Diagnostic criteria for polycystic ovary syndrome; towards a rational approach. In: Dunaif A, Givens JR, Haseltine F, editors. Polycystic ovary syndrome. Boston: Blackwell Scientific; 1992. p. 377-84.

121. Rotterdam ESHRE/ASRM-sponsored PCOS consensus workshop group. Revised 2003 consensus on diagnostic criteria and long-term health risks related to polycystic ovary syndrome (PCOS). Hum Reprod. 2004;19(1):41-7.

122. Azziz R, Carmina E, Dewailly D, Diamanti-Kandarakis E, Escobar-Morreale HF, Futterweit W, Witchel SF. The androgen excess and PCOS society criteria for the polycystic ovary syndrome: the complete task force report. Fertil Steril. 2008;91(2):456-88. 
123. Waters L, Reeves M, Fjeldsoe B, Eakin E. Control group improvements in physical activity intervention trials and possible explanatory factors: a systematic review. J Phys Act Health. 2012;9(6):884-95.

124. Cornelissen VA, Smart NA. Exercise training for blood pressure: a systematic review and meta-analysis. J Am Heart Assoc. 2013;2(1):1-9.

125. Domecq JP, Prutsky G, Mullan RJ, Hazem A, Sundaresh V, Elamin MB, Erwin P. Lifestyle modification programs in polycystic ovary syndrome: systematic review and meta-analysis. J Clin Endocrinol Metab. 2013;98(12):4655-63.

126. Moran LJ, Hutchison SK, Norman RJ, Teede HJ. Lifestyle changes in women with polycystic ovary syndrome. Cochrane Libr. 2011.

127. luorno MJ. Role of hyperinsulinemic insulin resistance in polycystic ovary syndrome. In: Kovacs GT, Norman R, editors. Polycystic ovary syndrome. 2nd ed. Cambridge: Cambridge University Press; 2007. p. 204-14.

128. Marin CM, Bartolucci A, Azziz R. Prevalence of insulin resistance in polycystic ovary syndrome (PCOS) patients using the homeostatic measurement assessment (HOMA-IR). Fertil Steril. 2003;80:274-5.

129. Dunaif A. Insulin resistance and the polycystic ovary syndrome: mechanism and implications for pathogenesis. Endocr Rev. 1997;18:774-800.

130. Chevenne D, Trivin F, Porquet D. Insulin assays and reference values. Diabetes Metab. 1999;26(6):459.

131. Boyns DR, Crossley JN, Abrams ME, Jarrett RJ, Keen H. Oral glucose tolerance and related factors in a normal population sample-l. Blood sugar, plasma insulin, glyceride, and cholesterol measurements and the effects of age and sex. Br Med J. 1969;1(5644):595-8.

132. Zhang HY, Guo CX, Zhu FF, Qu PP, Lin WJ, Xiong J. Clinical characteristics, metabolic features, and phenotype of Chinese women with polycystic ovary syndrome: a large-scale case-control study. Arch Gynecol Obstet. 2013; 287(3):525-31.

133. Olefsky J, Farquhar JW, Reaven GM. Relationship between fasting plasma insulin level and resistance to insulin-mediated glucose uptake in normal and diabetic subjects. Diabetes. 1973;22:507-13.

134. Philips DIW, Clark PM, Hales CN, Osmond C. Understanding oral glucose tolerance: comparison of glucose or insulin measurements during the oral glucose tolerance test with specific measurements of insulin resistance and insulin secretion. Diabetic Med. 1994;11:286-92.

135. Emoto M, Nishizawa Y, Maekawa K, Hiura Y, Kanda H, Kawagishi T, Morii H. Homeostasis model assessment as a clinical index of insulin resistance in type 2 diabetic patients treated with sulfonylureas. Diabetes Care. 1999; 22(5):818-22.

136. Ascaso JF, Pardo S, Real JT, Lorente RI, Priego A, Carmena R. Diagnosing insulin resistance by simple quantitative methods in subjects with normal glucose metabolism. Diabetes Care. 2003;26(12):3320-5

137. Haqq L, McFarlane J, Dieberg G, Smart N. The effect of lifestyle intervention on body composition, glycemic control, and cardiorespiratory fitness in polycystic ovarian syndrome: a systematic review and meta-analysis. Int J Sport Nutr Exerc Metab. 2015;25(6):533-40.

138. National Cholesterol Education Program. Second Report of the Expert Panel on Detection, Evaluation, and Treatment of High Blood Cholesterol in Adults, vol. 93. Maryland:NIH Publication; 1993. p. 3095.

139. Sniderman AD, Pedersen T, Kjekshus J. Putting low density lipoproteins at center stage in atherogenesis. Am J Cardiol. 1997;79:64-7.

140. National Institutes of Health Consensus Development Panel. Triglyceride, HDL, and CHD. JAMA. 1993:269:505-10.

141. Puska P. From Framingham to North Karelia: from descriptive epidemiology to public health action. Prog Cardiovasc Dis. 2010;53(1):15-20

142. Wadhera RK, Steen DL, Khan I, Giugliano RP, Foody JM. A review of low-density lipoprotein cholesterol, treatment strategies, and its impact on cardiovascular disease morbidity and mortality. J Clin Lipidol. 2016; 10(3):472-89.

143. Bass KM, Newschaffer CJ, Klag MJ, Bush TL. Plasma lipoprotein levels as predictors of cardiovascular death in women. Arch Intern Med. 1993;153(19): 2209-16.

144. Blair SN, Kohl HW, Paffenbarger RS, Clark DG, Cooper KH, Gibbons LW. Physical fitness and all-cause mortality. A prospective study of healthy men and women. JAMA. 1989;262(17):2395-401.

145. Kodama S, Saito K, Tanaka S, Maki M, Yachi Y, Asumi M, Sone H. Cardiorespiratory fitness as a quantitative predictor of all-cause mortality and cardiovascular events in healthy men and women: a meta-analysis. JAMA. 2009;301(19):2024-35.

146. Donà S, Bacchi $E_{1}$ Moghetti P. Is cardiorespiratory fitness impaired in PCOS women? A review of the literature. J Endocrinol Investig. 2017;40(5):463-69.
147. Orio F Jr, Giallauria F, Palomba S, Cascella T, Manguso F, Vuolo L, Russo T, Tolino A, Lombardi G, Colao A, Vigorito C. Cardiopulmonary impairment in young women with polycystic ovary syndrome. J Clin Endocrinol Metab. 2006:91:2967-71.

148. Bacchi E, Negri C, Di Sarra D, Tosi F, Tarperi C, Moretta R, Schena F, Bonora E, Kaufman JM, Moghetti P. Serum testosterone predicts cardiorespiratory fitness impairment in normal-weight women with polycystic ovary syndrome. Clin Endocrinol. 2015;83:895-901.

149. Lean MEJ, Han TS, Morrison CE. Waist circumference as a measure for indicating need for weight management. BMJ. 1995;311(6998):158-61.

150. Gómez-Ambrosi J, Silva C, Galofré JC, Escalada J, Santos S, Gil MJ, Frühbeck G. Body adiposity and type 2 diabetes: increased risk with a high body fat percentage even having a normal BMI. Obesity. 2011;19(7):1439-44.

151. Verweij LM, Terwee CB, Proper Kl, Hulshof CT, van Mechelen W. Measurement error of waist circumference: gaps in knowledge. Public Health Nutr. 2013;16(2):281-8.

152. Vanky E, Kjøtrød S, Salvesen KÅ, Romundstad P, Moen MH, Carlsen SM. Clinical, biochemical and ultrasonographic characteristics of Scandinavian women with PCOS. Acta Obstet Gyn Scan. 2004;83(5):482-6.

153. Enea C, Boisseau N, Fargeas-Gluck MA, Diaz V, Dugue B. Circulating androgens in women: exercise-induced changes. Sports Med. 2011;41(1):1-15.

154. Ennour-Idrissi K, Maunsell E, Diorio C. Effect of physical activity on sex hormones in women: a systematic review and meta-analysis of randomized controlled trials. Breast Cancer Res. 2015;17:139.

155. Tworoger SS, Missmer SA, Eliassen H, Barbieri RL, Dowsett M, Hankinson SE. Cancer Cause Control. 2007:18(7):743-52.

156. Kyrou I, Weickert MO, Randeva H. Diagnosis and management of PCOS. In: Ajjan R, editor. Endocrinology and diabetes: case studies, questions and commentaries. UK: springer; 2014. p. 9.
Ready to submit your research? Choose BMC and benefit from:

- fast, convenient online submission

- thorough peer review by experienced researchers in your field

- rapid publication on acceptance

- support for research data, including large and complex data types

- gold Open Access which fosters wider collaboration and increased citations

- maximum visibility for your research: over $100 \mathrm{M}$ website views per year

At $\mathrm{BMC}$, research is always in progress.

Learn more biomedcentral.com/submissions 